This item is the archived peer-reviewed author-version of:

\title{
Prognostic and predictive value of RAS gene mutations in colorectal cancer : moving beyond KRAS exon 2
}

\section{Reference:}

Boeckx Nele, Peeters Marc, Van Camp Guy, Pauwels Patrick, op de Beeck Ken, Deschoolmeester Vanessa.- Prognostic and predictive value of RAS gene mutations in colorectal cancer : moving beyond KRAS exon 2

Drugs - ISSN 0012-6667 - Northcote, Adis int Itd, 75:15(2015), p. 1739-1756

Full text (Publishers DOI): http://dx.doi.org/doi:10.1007/s40265-015-0459-x

To cite this reference: http://hdl.handle.net/10067/1294590151162165141 
1 Prognostic and Predictive Value of RAS Gene Mutations in Colorectal

2 Cancer: Moving Beyond KRAS Exon 2

3

4 Running title: Prognostic and Predictive Value of New RAS Mutations in

5 Colorectal Cancer

6

7 Nele Boeckx ${ }^{1,3}$, Marc Peeters ${ }^{1,2}$, Guy Van Camp ${ }^{3}$, Patrick Pauwels ${ }^{1,4}$, Ken Op de

8 Beeck $^{1,3^{*}}$, and Vanessa Deschoolmeester ${ }^{1,4^{*}}$

$9 \quad{ }^{1}$ Center for Oncological Research Antwerp, University of Antwerp, Antwerp, Belgium

$10{ }^{2}$ Department of Oncology, Antwerp University Hospital, Antwerp, Belgium

$11{ }^{3}$ Center for Medical Genetics, University of Antwerp, Antwerp University Hospital, Antwerp, Belgium

$12{ }^{4}$ Department of Pathology, Antwerp University Hospital, Antwerp, Belgium

13 *These authors contributed equally to this work

14 Contact information

Nele Boeckx, Campus Drie Eiken, Universiteitsplein 1, B-2610 Wilrijk, +32 3275 97 80, Nele.Boeckx@uantwerpen.be

Marc Peeters, UZA, Wilrijkstraat 10, B-2650 Edegem, Tel. +32 382139 54, Fax. +32 382141 21, Marc.Peeters@uza.be

Guy Van Camp, Campus Drie Eiken, Universiteitsplein 1, B-2610 Wilrijk, +32 327597 62, Guy.Vancamp@uantwerpen.be

Patrick Pauwels, UZA, Wilrijkstraat 10, B-2650 Edegem, Tel. +32 382137 53, Fax. +32 382147 53, Patrick.Pauwels@uza.be

Ken Op de Beeck, Campus Drie Eiken, Universiteitsplein 1, B-2610 Wilrijk, +32 327597 81, Ken.Opdebeeck@uantwerpen.be

Vanessa Deschoolmeester, Campus Drie Eiken, Universiteitsplein 1, B-2610 Wilrijk, +32 3265 25 76, Vanessa.Deschoolmeester@uantwerpen.be

22 Corresponding Author: Marc Peeters, UZA, Wilrijkstraat 10, B-2650 Edegem, Tel. +32 38213954, Fax. +32 382141

23 21, Marc.Peeters@uza.be 


\section{Abstract}

The advent of anti-EGFR therapy resulted in a significant progress in the treatment of metastatic colorectal cancer patients. However, many patients do not respond to this therapy or develop acquired resistance within a few months after the start of treatment. Since 2008, anti-EGFR therapy is restricted to KRAS wild-type patients as it has been shown that KRAS exon 2-mutated patients do not respond to this therapy. Still, up to $60 \%$ of KRAS exon 2 wild-type patients show primary resistance to this treatment. Recently, several studies investigating the predictive and prognostic role of RAS mutations other than in KRAS exon 2 demonstrated that patients with these mutations are not responding to therapy. However, the role of these mutations has long been questioned as The National Comprehensive Cancer Network Guidelines in Oncology and the European Medicines Agency indications had already been changed in order to restrict anti-EGFR therapy to all RAS wild-type colorectal cancer patients, while the Food and Drug Administration guidelines remained unchanged. Recently, the Food and Drug Administration guidelines have also been changed, which implies the importance of RAS mutations beyond KRAS exon 2 in colorectal cancer. In this review, we will discuss the most important studies regarding the predictive and prognostic role of RAS mutations other than in KRAS exon 2 in order to demonstrate the importance of these RAS mutations in patients with metastatic colorectal cancer treated with anti-EGFR therapy.

\section{$41 \quad$ Key Points}

42 RAS mutations, other than KRAS exon 2, are also responsible for resistance to anti-EGFR therapy in 43 patients with metastatic colorectal cancer.

Mutation-analysis on KRAS and NRAS exon 2 (codon 12 and 13), 3 (codon 59 and 61), and 4 (codon 117 and 146) on tumor tissue of mCRC patients is advised before starting anti-EGFR therapy with detection platforms that are sensitive enough to detect mutations at an allele frequency threshold of $\leq 5 \%$.

A lot of patients will benefit from extended RAS testing as they will no longer be exposed to unnecessary 50 toxicities and costs.

\section{1. Introduction}

52 Colorectal cancer (CRC) is a widespread type of cancer, characterized by high morbidity and mortality. It 53 is the second most commonly diagnosed cancer in females following breast cancer and the third in 
males, following lung and prostate cancer. In 2008, 608.700 CRC patients died and 1,2 million new CRC 55 patients were diagnosed worldwide [1].

56 CRC patients can be treated effectively or can even be cured by complete surgical resection of the 57 primary tumor and the local lymph nodes when the tumor is detected in its early stages. However, 58 surgery has limited efficacy when the tumor has spread to other organs. The 5-year survival rate of 59 patients with CRC detected and treated in its early stages is $90 \%[2,3]$. This rate drops to $10 \%$ in patients 60 with metastatic colorectal cancer ( $\mathrm{mCRC}$ ). At diagnosis, approximately $35 \%$ of patients have metastatic 61 disease and during the course of disease, $20 \%$ to $50 \%$ of stage II or III patients develop metastases [4]. A 62 subset of $\mathrm{mCRC}$ patients, with metastases limited to the liver and the lungs, can be cured with surgery, 63 preceded and/or followed by chemotherapy. However, in the vast majority of mCRC patients, surgery is 64 not curative [5].

65 Targeted therapies, such as cetuximab and panitumumab, have been developed for treatment of mCRC 66 patients. Cetuximab and panitumumab are both monoclonal antibodies directed against the epidermal 67 growth factor receptor (EGFR). Activation of the EGFR pathway in cancer cells has been linked to increased cell proliferation, angiogenesis, metastasis, and decreased apoptosis [6]. Inhibition of this pathway by anti-EGFR therapy has shown survival improvements of mCRC patients in several clinical trials [7-10].

71 Unfortunately, cetuximab and panitumumab are only effective in approximately $10 \%$ to $20 \%$ of 72 chemoresistant CRC patients [11-14]. Only a fraction of CRC patients respond to anti-EGFR therapy and 73 almost all responders become resistant after a few months of treatment [12, 13, 15]. In addition, this 74 therapy is costly and associated with potential harmful side effects, such as skin toxicity, neutropenia, 75 fatigue, nausea, vomiting, diarrhea, anorexia, constipation, and hypomagnesia $[15,16]$. Therefore, there 76 is a growing need for biomarkers that are able to identify patients who will respond to anti-EGFR therapy. A lot of research has already been performed on this theme in order to improve patient selection. A better selection can avoid unnecessary toxicities and costs in patients that will not respond. In addition, the survival and quality of life of these patients might increase, as other and potentially more effective therapies can be started earlier.

81 Since 2008, anti-EGFR therapy has been restricted to KRAS exon 2 wild-type (WT) patients, as it was 82 shown that KRAS exon 2-mutated patients do not respond to this therapy [7-10,17]. However, up to $60 \%$ 83 of these KRAS exon 2 WT patients are still resistant to anti-EGFR therapy [18, 19].

84 Recently, interesting results on anti-EGFR therapy in first-line setting as well as other lines of treatment 85 were published. In these studies, it has been shown that patients with mutations in KRAS, other than 
exon 2, and NRAS, the so-called new RAS mutations, did not respond to anti-EGFR therapy [20, 21].

87 Therefore, the European guidelines and the National Comprehensive Cancer Network Guidelines in 88 Oncology (NCCN) for both cetuximab and panitumumab have been revised to recommend that CRC patients with any KRAS or NRAS mutation should not be treated with eiter cetuximab or panitumumab $[11,22,23]$. Recently, the US Food and Drug Administration guidelines (FDA guidelines) have been adapted as well which means that all important agencies agree on this theme. In this review, we will summarize and discuss the most important studies that have investigated the significance of new RAS mutations in order to understand the real predictive and prognostic value of RAS mutations, beyond KRAS exon 2.

\section{EGFR pathway}

The epidermal growth factor receptor is a member of the EGFR family, a group of receptor tyrosine kinases, that mediate cell proliferation, survival, migration, and differentiation [24]. In addition to EGFR, the EGFR family has three other members: ERBB2 (formerly HER2/neu), ERBB3 (formerly HER3), and ERBB4. These receptor tyrosine kinases are transmembrane glycoproteins that exert their enzymatic activity in the cytoplasm. The receptors are inactive as single molecules but form activated homo- or heterodimers when a ligand binds to the extracellular ligand-binding domain of the receptor. In this way, the receptors can translate extracellular signals into intracellular activity [6, 25]. EGFR is frequently overexpressed and activated in colorectal tumors, and therefore a possible target in CRC treatment [16, $26,27]$.

EGFR is activated by binding a ligand, such as epidermal growth factor (EGF), transforming growth factor$\alpha$, amphiregulin, and epiregulin to its extracellular domain [6]. EGFR exerts its activity via two main pathways, the RAS/RAF/MAPK (mitogen-activated protein kinase) pathway and the (phosphatidylinositol-3-kinase) PI3K/AKT pathway (Figure 1) [28, 29]. In the RAS/RAF/MAPK pathway, receptor dimerization due to ligand-binding leads to the activation of RAS [30]. The RAS family is encoded by three genes: HRAS, NRAS, and KRAS [31]. The RAS proteins are small guanine nucleotide-binding proteins (GTPases) that act as intracellular signal transducers. These proteins transduce extracellular signals to the cytosol and the nucleus leading to the activation of different transcription factors [3, 32]. RAS proteins show a spontaneous dephosphorylation activity and can be present in two different states, the active guanosine triphosphate (GTP)-bound state and the inactive guanosine diphosphate (GDP)-bound state. Switching between both states is supported by regulatory proteins, such as RAS guanine nucleotide exchange factors (GEFs) and RAS GTPase-activating proteins (GAPs), as the intrinsic GTPase-activity of RAS is very slow. The function of GEFs is the activation 
of RAS by releasing GDP out of the GDP-RAS complex. This release creates a binding opportunity for GTP, which is present in high concentration in the cytoplasm. The inactivation of RAS on the other hand is supported by GAPs, which stimulate the dephosphorylation of GTP [3, 33]. Active GTP-bound RAS recruits the serine-threonine protein kinase BRAF to the cell membrane which results in the activation of BRAF [30]. Afterwards, BRAF activates mitogen-activated protein kinase kinase (MAP2K or MEK), which induces the activation of MAPK. Finally, MAPK activates transcription factors that are involved in cell proliferation, survival, growth, angiogenesis, and motility [3]. In the PI3K/AKT pathway, ligand-binding to EGFR leads to the activation of PIK3CA, which phosphorylates phosphatidylinositol-4-5-biphospate (PIP2) to phosphatidylinositol-3,4,5-triphosphate (PIP3). Next, PIP3 activates AKT resulting in the phosphorylation of downstream protein effectors, including mTOR. The pathway is negatively regulated by PTEN, which dephosphorylates PIP3 to PIP2 [34, 35].

\section{Current guidelines regarding the use of anti-EGFR therapy}

Cetuximab, a mouse chimeric monoclonal antibody and panitumumab, a fully human monoclonal antibody are both directed against the EGFR pathway. These antibodies target the extracellular ligandbinding domain of the receptor, which prevents ligand-binding and receptor dimerization. This results in the inhibition of ligand-induced cell survival, cell growth, cell proliferation, and angiogenesis (Figure 1) [3].

Both monoclonal antibodies have been reported to be effective as single agents and in combination with chemotherapy for mCRC treatment, as shown in different clinical trials by improved progression-free survival (PFS), response rate (RR) or quality of life [12-14, 36]. In 2004 and 2006 respectively, cetuximab and panitumumab were approved for the treatment of $\mathrm{MCRC}$ patients [37]. However, it was clear that more research on predictive markers was needed, as many patients did not respond to anti-EGFR therapy.

In 2006, Lièvre et al. were the first to report that CRC patients carrying a mutation in KRAS exon 2 showed resistance to anti-EGFR therapy [8]. Since then, rapidly accumulating publications of clinical trials provided compelling evidence that patients with KRAS mutations in codon 12 or 13 of exon 2 are resistant to cetuximab and panitumumab $[7,9,10,13,17,38-45]$.

Codon 12 and 13 encode two adjacent glycine residues that are located in the proximity of the catalytic site of KRAS. Mutations in this area cause a reduction in the intrinsic GTPase activity, impeding the normal inactivation of KRAS [46]. These mutations result in constitutively activated KRAS and consequently in a constitutive activation of the RAS/RAF/MAPK pathway, even in the presence of cetuximab or panitumumab, or in the absence of a ligand that binds to $\operatorname{EGFR}[10,15]$. 
In 2009, the American Society of Clinical Oncology (ASCO) released provisional guidelines recommending restriction of anti-EGFR therapy to patients with KRAS exon 2 WT tumors. It was advised to analyze the mutation status of KRAS codon 12 and 13 in all candidate patients for anti-EGFR therapy. If a mutation was detected in one of these codons, patients should not receive anti-EGFR therapy [18]. Subsequently, the FDA indications for anti-EGFR therapy were also changed reflecting the same guidelines [47]. During the last five years, there has been some debate about the predictive value of the KRAS G13D mutation, as some authors have reported that patients with this mutation might respond to anti-EGFR therapy. However, there are still some doubts about the real predictive value of this mutation (see section 4 . Predictive value of the KRAS G13D mutation in patients receiving anti-EGFR therapy). Therefore, the guidelines have not been altered and anti-EGFR therapy is still restricted to patients that have no mutations in KRAS codon 12 and 13. Unfortunately, not all KRAS exon 2 WT patients respond to anti-EGFR therapy. A lot of research has been performed in order to study the effect of mutations in genes encoding other proteins involved in the been published on the mutation status of NRAS and KRAS (beyond exon 2). The conclusion of these new data holds that patients with NRAS and rare KRAS mutations do not respond to anti-EGFR therapy [20, 21]. Due to these findings, the NCCN Guidelines for CRC have been adapted. Currently, the NCCN Guidelines recommend that all MCRC patients should have either primary or metastatic tumor tissue tested for KRAS and NRAS mutations [11]. Patients harboring any RAS mutation cannot be treated with anti-EGFR therapy. In addition, the European Medicines Agency (EMA) and FDA indications for anti-EGFR therapy have been updated to restrict the therapy to all RAS WT patients [22, 23, 51, 52]. In the following part, the most important studies, assessing the role of new RAS mutations in mCRC patients treated with anti-EGFR therapy will be summarized. In addition, the predictive value of the KRAS G13D mutation will be discussed, as there is still some debate about the role of this mutation.

\section{Predictive value of the KRAS G13D mutation in patients receiving anti-EGFR}

\section{therapy}

176 Although the mutation-analysis in KRAS exon 2 (codon 12 and 13 ) is performed before starting anti-EGFR therapy today, there is still some discussion about the effect of the glycine to aspartate mutation at codon 13 (G13D) in KRAS on clinical outcomes in patients treated with anti-EGFR therapy. Contrasting results have been published on this topic. from anti-EGFR monoclonal antibody therapy in chemotherapy-refractory settings and in first-line 
combination therapy with irinotecan or oxaliplatin $[53,54]$. De Roock et al. (2010) compared the outcomes of patients with the KRAS G13D mutation to those of patients with other KRAS mutations among patients with chemotherapy-refractory mCRC treated with cetuximab. Thirty-two patients harbored the KRAS G13D mutation. These patients had longer PFS (4.0 vs. 1.9 months, $p=0.004)$ and overall survival (OS) (7.6 vs. 5.7 months, $\mathrm{p}=0.005)$ than patients with other KRAS mutations, but no significant difference in $\mathrm{RR}(6.3 \%$ vs. $1.6 \%, \mathrm{p}=0.19)$ was seen. In addition, there was no significant difference in PFS (4.0 vs. 4.2 months, $p=0.66$ ) and OS (7.6 vs. 10.1 months, $p=0.79$ ) between KRAS G13Dmutated and KRAS WT patients, but there was a significant difference in RR (6.3\% vs. $26.4 \%, p=0.02)$. The authors believe that patients with KRAS G13D-mutated tumors respond to cetuximab therapy, but with a lower RR than KRAS WT patients. In addition, De Roock et al. performed an in vitro and in vivo mouse model analysis that showed that KRAS G13D-mutated colorectal cancer cells were sensitive to cetuximab, similar to KRAS WT cells, while KRAS G12V-mutated colorectal cancer cells were insensitive. It was concluded that the prolonged PFS and OS of KRAS G13D-mutated patients compared to patients with other KRAS mutations was due to a delay in progression but not to a real reduction in tumor burden. A possible explanation is that the proliferation of tumor cells is inhibited (cytostatic effect) on EGFR inhibition instead of undergoing apoptosis (cytotoxic effect). However, it should be taken into account that the response rate of KRAS G13D-mutated patients to cetuximab monotherapy was very low. None of these patients responded to monotherapy. This might indicate that the responses that were seen in the cetuximab plus chemotherapy group may reflect responsiveness to chemotherapy rather than to cetuximab [53].

Tejpar et al. (2012) investigated the association between the KRAS mutation status (WT, G13D, G12V, or other mutations) and PFS, OS, and response in pooled data from the CRYSTAL (Cetuximab Combined With Irinotecan in First-line Therapy for Metastatic Colorectal Cancer) and OPUS (Oxaliplatin and Cetuximab in First-line Treatment of Metastatic Colorectal Cancer) studies. Out of 1378 eligible patients, they found 533 patients (39\%) with mutations in KRAS. G13D, G12V, and other mutations were found in 83 (16\%), 125 (23\%), and 325 (61\%) patients, respectively. By comparing patients with the KRAS G13D mutation to patients harboring all other KRAS mutations (including G12V), significant variations were found in tumor response $(\mathrm{p}=0.005)$ and PFS $(\mathrm{p}=0.046)$. Among all KRAS G13D-mutated patients, the addition of cetuximab to chemotherapy compared to chemotherapy alone resulted in a significantly improved PFS (7.4 vs. 6.0 months, $p=0.039)$ and RR (40.5\% vs. $22.0 \%, p=0.042)$. However, no improvement in OS was seen (15.4 vs. 14.7 months, $\mathrm{p}=0.68$ ). Contrary to KRAS G13D-mutated patients, patients with other RAS mutations did not benefit from the addition of cetuximab. Furthermore, the 

than patients harboring other RAS mutations. Tejpar et al. concluded that the addition of cetuximab was

216 beneficial for patients with the KRAS G13D mutation in first-line treatment with chemotherapy. The 217 observed positive treatment effect for these patients was caused by the combination of the poor 218 prognosis observed when those patients received only chemotherapy, and the improved outcome under 219 treatment with cetuximab [54]. Other studies performed by Benvenuti et al. (2007) and Molinari et al. 220 (2011) reported partial response in 1 out of 6 and 2 out of 11 KRAS G13D-mutated patients, respectively $221[17,55]$.

222 Next to these clinical trials, some in vivo studies have shown similar results. Preclinical studies have 223 suggested that individual KRAS codon 12 or 13 alleles show quantitative and/or qualitative differences in 224 transforming capacity and other biologic phenotypes. Specifically, in comparison to KRAS codon 13 225 mutations, KRAS codon 12 mutations seem to have greater in vitro transforming ability [56-58].

226 Furthermore, Alamo et al. (2014) recently showed by injecting recombinant clones of the SW48 CRC cell 227 line expressing the KRAS G12V mutation or the KRAS G13D mutation in mice cecum that KRAS G12V 228 mutations have a higher metastatic potential than KRAS G13D mutations [59].

229 Contrary to these results, Peeters et al. (2013) found that patients with mutations in KRAS codon 12 or 23013 do not benefit from panitumumab therapy. A retrospective analysis of three randomized phase III 231 studies was performed in order to assess the prognostic and predictive impact of these mutations on 232 survival outcomes in 1053 mCRC patients. None of the individual mutant KRAS alleles were consistently 233 associated with panitumumab treatment effect on PFS or OS outcomes, across the three studies. 234 However, the collective group of mutant KRAS alleles was a negative predictive factor for both PFS and 235 OS in therapies containing panitumumab. The authors concluded that patients with KRAS codon 12 or 13 236 mutations are unlikely to benefit from panitumumab treatment [41].

237 Furthermore, Schirripa et al. (2014) conducted a prospective trial in order to confirm the 238 aforementioned findings of De Roock et al. and Tejpar et al. and in order to evaluate the clinical 239 relevance of cetuximab in KRAS G13D-mutated patients. Therefore, 12 KRAS G13D-mutated mCRC 240 patients treated with cetuximab monotherapy were enrolled. If only three or less of these patients 241 would have been progression-free at four months after treatment start, the hypothesis that KRAS G13D242 mutated patients experience benefit from cetuximab would have been rejected. At four months after 243 treatment start, it was found that three patients (25\%) showed disease stabilization and that no RECIST 244 responses were observed. The authors concluded that there was no clinically relevant benefit with 
cetuximab monotherapy in KRAS G13D-mutated mCRC patients and that these patients should not be

246 treated with cetuximab [60].

247 In addition, a recent retrospective analysis of 110 patients treated with cetuximab, was performed by 248 Gajate et al. (2012). They reported that patients with the KRAS G13D mutation did not benefit from 249 cetuximab treatment. In these patients, a trend towards lower OS was detected compared to KRAS WT 250 patients or patients with other KRAS mutations [61].

251 In conclusion, there is still no consensus about the predictive value of the KRAS G13D mutation. As long 252 as there is no clear evidence of tumor response in KRAS G13D-mutated patients, the guidelines will 253 remain unchanged.

\section{Predictive value of new RAS mutations in patients receiving anti-EGFR therapy}

255

256

During the last decade, a lot of research has been performed on the predictive potential of the RAS mutation status in $\mathrm{mCRC}$ patients treated with anti-EGFR therapy. We report the most important studies according to the monoclonal antibody that has been used in the different clinical trials.

258 The frequency of all new RAS mutations per study are reported in table 1 . In addition, the response rates 259 in the different studies and the survival outcomes are shown in table 2 and 3, respectively. In general, all studies support a negative predictive role of new RAS mutations in patients treated with anti-EGFR 261 therapy.

\subsection{Cetuximab-based therapy}

263 Loupakis et al. (2009) were one of the first to study KRAS mutations outside exon 2. They investigated 264 the role of mutations in KRAS codon 61 (exon 3) and 146 (exon 4) regarding resistance to cetuximab plus irinotecan in a cohort of patients with no mutations in KRAS codon 12 and 13 . Seven patients (8\%) with a mutation in KRAS codon 61 and 1 patient (1\%) with a mutation in KRAS codon 146 were identified. None of these patients showed response to therapy while 22 of 68 WT KRAS patients did respond $(p=0.096)$. In addition, patients with mutations in codon 61 and 146 had a significantly shorter PFS than KRAS WT patients (3.8 vs. 5.1 months, $p=0.028$ ). However, no significant difference in OS was reported (9.7 vs. 14.7 months, $p=0.390$ ), which may be explained by the low amount of mutated cases [62].

272 of cetuximab in patients with chemotherapy-refractory mCRC treated with chemotherapy plus 273 cetuximab. KRAS mutations were detected in $40 \%$ of patients (299/747), including 36.3\% in exon 2 274 (codon 12/13), 2.1\% in codon 61, and $2.0 \%$ in codon 146. They also detected one patient with a KRAS 275 mutation in codon 59. Double KRAS mutations were detected in four tumors (G12V+G12S, A146T+Q61L, 
and twice G12V+A146T). KRAS-mutated patients had a significantly lower RR than KRAS WT patients

277 (6.7\% (17/253) vs. 35.8\% (126/352), p<0.0001). In addition, they had a shorter median PFS (12 vs. 24 weeks, $\mathrm{p}<0.0001$ ) and OS (32 vs. 50 weeks, $\mathrm{p}<0.0001)$. Among the evaluable KRAS-mutated patients, 13 patients had a mutation in codon 61. Compared to KRAS WT patients, a significantly lower $R R(p=0.0055)$ was seen in KRAS codon 61-mutated patients, as none of them responded to therapy. In contrast to these findings, there was no significant difference in RR between patients with mutations in KRAS codon 146 and KRAS WT patients ( $\mathrm{p}=0.34)$, as 2 out of 11 patients (18.2\%) with KRAS codon 146 mutations did respond to therapy. Although the number of KRAS-mutated patients was low, these results indicate that patients with KRAS codon 61 mutations do not respond to anti-EGFR therapy, while patients with codon 146 mutations might still respond. In addition, De Roock et al. detected NRAS mutations in $2.6 \%$ of KRAS WT patients (17/644). Most of them occurred in codon 61, rather than in codon 12 or 13 . These patients had a significantly lower RR compared to NRAS WT patients $(7.7 \%(1 / 13)$ vs. $38.1 \%(110 / 289), p=0.013)$ and a trend towards shorter median PFS ( 14 vs. 26 weeks, $p=0.055$ ) and OS ( 38 vs. 50 weeks, $p=0.051$ ). These results show the inefficacy of cetuximab in NRAS-mutated mCRC patients. Since the number of NRAS-mutated patients is low, the effect on survival is not clear [48].

Heinemann et al. (2014) recently published the results of the re-analysis of the FIRE-3 study, a randomized, open-label, phase III trial. This study was originally designed in order to compare the objective response in KRAS exon 2 WT mCRC patients treated with FOLFIRI plus cetuximab vs. FOLFIRI plus bevacizumab. In this subgroup of patients, there was no significant difference in objective RR (62.0\% vs. $58.0 \%, p=0.18)$ and median PFS (10.0 vs. 10.3 months, $p=0.55)$ in both treatment arms. However, the median OS was significantly longer in the FOLFIRI plus cetuximab arm vs. the FOLFIRI plus bevacizumab arm ( 28.7 vs. 25 months, $\mathrm{p}=0.017$ ). For the re-analysis, the tumor mutation status of KRAS exon 3 (codon 61), exon 4 (codon 146) and NRAS exon 2 (codon 12 and 13), exon 3 (codon 59 and 61), and exon 4 (codon 117 and 146) was assessed using pyrosequencing in 407 evaluable patients. New RAS mutations were detected in 65 patients (16\%). Similar results were obtained for the objective response (65\% vs. $60 \%, p=0.32$ ) and PFS (10.4 vs. 10.2 months, $p=0.54)$ when comparing the cetuximab arm to the bevacizumab arm in patients with all RAS WT tumors. However, there was a marked advantage in OS for patients treated with FOLFIRI plus cetuximab (33.1 vs. 25.6 months, $p=0.011$ ). Several possible explanations exist for the fact that these patients experience an OS benefit without a difference in PFS or RR. First, some bias can be present in response assessments as there was no independent radiological review of the response data. Further, response to therapy might not be captured adequately by RECIST criteria when using different strategies of targeted therapy. Last, although the number of patients that 
received second-line therapy was similar in each treatment group, and although the number of patients that crossed over to the alternative anti-VEGF or anti-EGFR therapy was similar, the sequence of targeted agents for patients in the treatment groups was in many cases reversed. This can lead to changes in tumor biology during first-line therapy, which might be related to the difference in OS between both treatment groups. In experimental models, the upregulation of VEGF in association with resistance to cetuximab has been reported [63-65]. Such phenotypic changes could benefit second-line anti-VEGF treatment after first-line cetuximab therapy. In the subgroup of patients that were WT for KRAS exon 2 but mutant for other RAS mutations, PFS was significantly lower in the cetuximab arm vs. the bevacizumab arm (6.1 vs. 12.2 months, $p=0.004)$, but the difference in $R R(38 \%$ vs. $58 \%, p=0.14)$ and OS (16.4 vs. 20.6 months, $p=0.57$ ) did not reach statistical significance. This study confirms that excluding all RAS-mutated patients for anti-EGFR therapy will lead to a population that is more likely to benefit from cetuximab and that the addition of cetuximab to RAS-mutated patients might have detrimental effects [63].

Recently, a re-analysis of the OPUS study was performed. In this study, mCRC patients were randomized to oxaliplatin, fluorouracil (FU), and leucovorin (FOLFOX4) treatment with or without cetuximab for firstline treatment. It was found that patients with mutations in KRAS exon 2 had no benefit from the addition of cetuximab, while the addition of cetuximab significantly improved PFS (8.3 vs. 7.2 months, $\mathrm{p}=0.0064)$ and RR ( $57 \%$ vs. $34 \%, \mathrm{p}=0.0027)$ in KRAS exon 2 WT patients. Results for OS were improved but did not reach statistical significance ( 22.8 vs. 18.5 months, $p=0.39$ ) [66]. Now, beads, emulsion, amplification, and magnetics technology (BEAMing) was used in order to screen for mutations in KRAS exon 3 and 4, and NRAS exon 2, 3, and 4 in patients with KRAS exon 2 WT tumors. New RAS mutations were found in $26 \%$ of patients (31/118). The addition of cetuximab to FOLFOX4 significantly improved RR (57.9\% vs. $28.6 \%, \mathrm{p}=0.008)$ and PFS ( 12.0 vs. 5.8 months, $\mathrm{p}=0.062)$ in all RAS WT patients, but there was no significant difference in OS (19.8 vs. 17.8 months, $p=0.8$ ). The lack of OS benefit in this group might be explained by the small sample size. Furthermore, the OPUS study is a phase II study where efficacy is a primary endpoint instead of OS. As the number of patients in the group of new RAS-mutated patients was low, treatment effect could not be assessed. However, no benefit was seen from the addition of cetuximab to FOLFOX4 in RR ( $37.0 \%$ vs. $50.7 \%, p=0.087)$ and PFS ( 5.6 vs. 7.8 months, $p=0.031$ ) in patients with any mutation in RAS. There was even a trend for worse outcome in the cetuximab arm (OS: 13.5 vs. 17.8 months, $p=0.157$ ) [67].

Van Cutsem et al. (2015) showed updated results of the phase III CRYSTAL trial. This study originally showed that patients with KRAS exon 2 WT tumors benefit from the addition of cetuximab to FOLFIRI in 
first-line treatment, evidenced by significantly improved PFS (9.9 vs. 8.4 months, $p=0.0012$ ), OS (23.5 vs. 20.0 months, $\mathrm{p}=0.0093)$, and RR $(57.3 \%$ vs. $39.7 \%, p<0.001)[38,68]$. Recently, the KRAS exon 2 WT patients were re-analyzed using BEAMing in order to find out whether these patients have mutations in KRAS exon 3 (codon 59 and 61) and exon 4 (codon 117 and 146), and in NRAS exon 2 (codon 12 and 13), exon 3 (codon 59 and 61), and exon 4 (codon 117 and 146). New RAS mutations were detected in 15\% of patients (63/430). In the subgroup of all RAS WT patients, a significant improvement in RR (66.3\% vs. $38.6 \%, p<0.001)$, PFS (11.4 vs. 8.4 months, $p<0.001)$, and OS ( 28.4 vs. 20.2 months, $p=0.0024$ ) was seen when cetuximab was added to FOLFIRI compared to FOLFIRI alone. Contrary to these results, no difference between both treatment arms was seen in RR (34.4\% vs. $35.5 \%, p=0.97)$, PFS (7.2 vs. 6.9 months, $\mathrm{p}=0.56)$, and OS (18.2 vs. 20.7 months, $\mathrm{p}=0.50$ ) in the group of patients with new RAS mutations $[69,70]$.

In sum, many studies indicate that patients with new RAS mutations do not benefit from cetuximab, while all RAS WT patients are susceptible to cetuximab, evidenced by improved response and survival outcomes. Furthermore, the addition of cetuximab to RAS-mutated patients might even be detrimental and consequently should be avoided.

\subsection{Panitumumab-based therapy}

In 2013, Peeters et al. analyzed 320 samples for mutations in nine genes (KRAS (codon 61), NRAS (codon 12, 13, and 61), BRAF, PIK3CA, PTEN, TP53, EGFR, AKT1, and CTNNB1) using massively parallel multigene sequencing in a randomized phase III study of $\mathrm{mCRC}$ in order to evaluate whether these mutations predicted response to panitumumab monotherapy. They reported that 1 out of 6 patients with mutations in KRAS codon 61 showed partial response. In addition, NRAS mutations were detected in 5\% of patients $(\mathrm{n}=14), 3$ of them had mutations in both KRAS and NRAS. None of the NRAS-mutated patients responded to panitumumab, while $17 \%$ of NRAS WT patients did respond to therapy. Furthermore, among KRAS and NRAS WT patients ( $\mathrm{n}=138)$, treatment with panitumumab compared to best supportive care was associated with improved PFS $(p<0.001)$, while panitumumab treatment was no longer associated with improved PFS ( $\mathrm{p}=0.379)$ in KRAS WT and mutant NRAS patients $(\mathrm{n}=11)$ [21].

Douillard et al. (2013) performed a prospective-retrospective analysis of the treatment effect of RAS (KRAS and NRAS) and BRAF mutations on PFS and OS in a randomized phase III study of panitumumab plus FOLFOX4 compared to FOLFOX4 alone in patients with previously untreated mCRC. Therefore, KRAS exon 2 WT patients of the PRIME trial (Panitumumab Randomized Trial in Combination with Chemotherapy for Metastatic Colorectal Cancer to Determine Efficacy) were re-analyzed. These patients were screened for mutations in KRAS exon 3 (codon 61) and exon 4 (codon 117 and 146), NRAS exon 2 
372 (codon 12 and 13), exon 3 (codon 61), and exon 4 (codon 117 and 146), and BRAF exon 15 (codon 600).

373 Among the 620 patients that were originally categorized as not having mutations in KRAS exon 2, 108

374 patients (17\%) harbored new RAS mutations. There were 24 patients with mutations in KRAS exon 3, 36

375 KRAS exon 4-mutated patients, 22 NRAS exon 2-mutated patients, and 26 NRAS exon 3-mutated

376 patients. No mutations were found in NRAS exon 4. Comparing the panitumumab arm to FOLFOX4 alone

377 in this group of 108 new RAS-mutated patients, there seemed to be a negative treatment effect of 378 panitumumab plus FOLFOX4 on PFS (7.3 vs. 8.0 months, $p=0.33$ ) and OS (17.1 vs. 17.8 months, $p=0.12$ )

379 but these results did not reach statistical significance. However, in the group of patients with any mutation in RAS, the survival outcomes were significantly worse in the panitumumab arm than in the FOLFOX4 arm (PFS: 7.3 vs. 8.7 months, $p=0.008$; OS: 15.5 vs 18.7 months, $p=0.04$ ), clearly showing a detrimental effect of adding panitumumab to first-line FOLFOX4 in patients with mutations in RAS. These results were comparable to those in the subgroup of KRAS exon 2-mutated patients (PFS: 7.3 vs. 8.8 months, $\mathrm{p}=0.02$; OS: 15.5 vs. 19.2 months, $\mathrm{p}=0.16$ ). Contrary to these findings, among the RAS WT patients $(n=512)$, a significant improvement in both PFS (10.1 vs. 7.9 months, $p=0.004)$ and OS ( 25.8 vs. 20.2 months, $p=0.009$ ) was detected in the panitumumab plus FOLFOX4 arm compared to FOLFOX4 alone. This finding confirms the positive effect of panitumumab in RAS WT patients. The authors concluded that all tested RAS mutations were negative predictive factors for treating $\mathrm{mCRC}$ patients with anti-EGFR therapy, as patients with mutant RAS tumors did not benefit from panitumumab treatment. Moreover, the addition of panitumumab to RAS-mutated patients was even detrimental [20]. Recently, the final results of PFS and OS from PRIME were published, 30 months after the last patient was enrolled. These results were similar to those described above [71]. André et al. (2013) performed a single-arm multicenter, phase II study in order to evaluate the efficacy and safety of the combination of irinotecan and panitumumab in KRAS WT mCRC patients that were heavily pretreated. In addition, this study explored other potential predictive genetic alterations. 396 Therefore, KRAS exon 2 WT patients were further screened for mutations in KRAS codon 59, 61, 117, and 146, in NRAS codon 12, 13, and 61, and in BRAF codon 600 using direct sequencing. Among 60 patients, 6 patients had a KRAS mutation in codon 12. This finding was surprising, as only patients with a KRAS codon 12 and 13 WT tumor were included in the study based on local molecular determination of the 400 mutational status performed in routine diagnosis. Central analysis using an allelic discrimination strategy 401 based on TaqMan mutation-specific probes for KRAS screening revealed discrepancies between laboratories. These discrepancies might be due to other testing methodologies or differences in expertise. Beside these KRAS codon 12 mutated patients, 4 patients had rare KRAS mutations (1 in codon 
59 and 3 in codon 61), 5 patients had NRAS mutations ( 1 in codon 12, 1 in codon 13, and 3 in codon 61), and 4 patients had BRAF mutations. None of them responded to therapy. Among the original group of KRAS exon 2 WT patients, the overall RR was $29.2 \%$, PFS was 5.5 months, and OS was 9.7 months. All parameters seemed to be improved in the subgroup of patients without mutations in KRAS, NRAS, and $B R A F$, as the RR was $46.3 \%$, PFS was 8.7 months, and OS was 15.8 months. Contrary to these results, a drop in all parameters was seen in the subgroup of mutated patients. RR was 0\%, PFS was 1.9 months, and OS was 4.6 months. These results confirm that RAS-mutated patients do not respond to anti-EGFR therapy. Moreover, excluding all RAS-mutated patients will lead to a population that is more likely to benefit from anti-EGFR therapy [72].

Patterson et al. (2013) published some additional results of the randomized, phase III mCRC study (20020408). In this monotherapy study, the addition of panitumumab was compared to best supportive care. It had been shown that patients with mutations in KRAS exon 3 and NRAS exon 2 and 3 did not benefit from the addition of panitumumab [21]. These results were expanded in order to study the effect of KRAS and NRAS mutations in exon 4. Of a total amount of 243 KRAS exon 2 WT patients, 9 and 2 patients harbored mutations in KRAS and NRAS exon 4, respectively. They also discovered one patient with mutations in both KRAS and NRAS exon 4. Among 95 KRAS/NRAS-mutated patients in the panitumumab arm, there was 1 mutant KRAS exon 4 patient that showed partial response. The overall RR of patients with mutations in KRAS or NRAS was $1 \%$ in the panitumumab arm, while the overall RR was $15 \%$ in patients with KRAS and NRAS WT tumors. In the best supportive care arm, no responses were shown. The authors concluded that patients with mutations in exon 4 of both KRAS and NRAS did not benefit from panitumumab therapy. Furthermore, patients with mutations in RAS exon 4 need to be excluded before starting anti-EGFR therapy despite the fact that these mutations are rare [73].

Schwartzberg et al. (2014) performed an extended RAS mutation-analysis on patients of the PEAK study (Panitumumab Efficacy in Combination With mFOLFOX6 against Bevacizumab Plus mFOLFOX6 in mCRC Subjects With Wild-Type KRAS tumors). The PEAK study is a phase II, open-label randomized study originally designed to estimate the effect of panitumumab in combination with modified FU, leucovorin, and oxaliplatin (mFOLFOX6) relative to bevacizumab plus mFOLFOX6 as first-line therapy in patients with KRAS exon 2 WT mCRC. In the KRAS exon 2 WT group, PFS was similar in both arms (10.9 months in the panitumumab arm vs. 10.1 months in the bevacizumab arm, $p=0.353$ ), while OS was significantly longer in the panitumumab arm compared to the bevacizumab arm (34.2 vs. 24.3 months, $p=0.009$ ). A secondary objective of this study was to assess PFS and OS in patients with RAS WT MCRC. Therefore an extended RAS analysis of exon 2 (codon 12 and 13), exon 3 (codon 59 and 61), and exon 4 (codon 117 
and 146) in both KRAS and NRAS was performed using real-time quantitative PCR in the central laboratory or other validated assays in local laboratories. New RAS mutations were detected in 51 of 221 patients (23\%). KRAS exon 3 and 4 mutations were detected in 9 and 17 patients and NRAS exon 2, 3, and 4 mutations were found in 12, 13 and 0 patients, respectively. In the all RAS WT group, PFS was improved in the panitumumab arm compared to the bevacizumab arm (13.0 vs. 9.5 months, p=0.029) and there was a trend for an improvement in OS (41.3 vs. 28.9 months, $\mathrm{p}=0.058$ ). In patients with KRAS exon 2 WT tumors that did have other RAS mutations, PFS seemed to be worse in the panitumumab arm compared to the bevacizumab arm, although these results were not statistically different ( 7.8 vs. 8.9 months, $p=0.318)$. OS, on the other hand, was improved in the panitumumab arm ( $27.0 \mathrm{vs.} 16.6$ months, $p=0.020$ ). This surprising result for OS can be explained by the relatively high percentage of patients in the panitumumab arm that received subsequent chemotherapy (83\%) and anti-VEGF therapy (53\%). In sum, this study confirms that only patients with RAS WT tumors benefit from anti-EGFR therapy and that these patients have more benefit from anti-EGFR therapy than anti-VEGF therapy in combination with mFOLFOX6 [74].

Peeters et al. (2014) reported new results on the phase III study 20050181. This study was originally designed in order to assess the effect on PFS and OS of panitumumab plus FOLFIRI vs. FOLFIRI alone in second-line treatment of $\mathrm{mCRC}$ patients. A significant improvement in PFS (6.7 vs. 4.9 months, $p=0.023$ ) and a trend towards improved OS (14.5 vs. 12.5 months, $p=0.37$ ) were detected in the panitumumab arm in KRAS exon 2 WT patients [7, 75]. Recently, a re-analysis was performed on the group of KRAS exon 2 WT patients. Mutations in KRAS exon 3 and 4 and in NRAS exon 2, 3, and 4 were investigated by bidirectional Sanger sequencing. New RAS mutations were found in $18 \%$ of the KRAS exon 2 WT patients (107/597). In the all RAS WT group, better PFS results (6.4 vs. 4.6 months, $\mathrm{p}=0.007$ ) and a trend towards improved OS (16.2 vs. 13.9 months, $p=0.08$ ) were found in the panitumumab plus FOLFIRI arm vs. FOLFIRI alone. No benefit could be detected from the addition of panitumumab to FOLFIRI in RASmutated patients for PFS (4.8 vs. 4.0 months, $p=0.14$ ) and OS (11.8 vs. 11.1 months, $p=0.34$ ). The authors found that similar to patients with mutations in KRAS exon 2, RAS-mutated patients are unlikely to benefit from the addition of panitumumab to FOLFIRI [76].

\subsection{Cetuximab- or panitumumab-based therapy}

Molinari et al. (2011) evaluated retrospectively the objective tumor responses in 111 evaluable mCRC patients that were treated with cetuximab- or panitumumab-based regimens. KRAS codon 12, 13, and 61 were analyzed for mutations. KRAS exon 2 mutations were found in 43 cases (39\%). Most of them occurred in codon 12 (31 cases, 74\%) and 11 cases (26\%) showed a mutation in codon 13. One patient 
showed KRAS mutations in both codon 12 and 13. In addition, KRAS exon 3 mutations were detected in 4 cases (4\%), including Q61H (2 cases), Q61L, and G60D. The G60D-mutated patient and 2 KRAS G13Dmutated patients did respond to cetuximab- or panitumumab-based therapy. Three patients were not evaluable for mutations in exon 3 due to a lack of material. The 3 KRAS codon 61-mutated patients showed progression of disease, but these mutations occurred concomitantly with other mutations. Therefore, the predictive value of KRAS codon 61 mutations could not be determined [55]. A meta-analysis was recently performed by Sorich et al. (2015) to investigate whether new RAS mutations are predictive for resistance to anti-EGFR therapy. The analysis was based on eight randomized controlled trials. New RAS mutations were detected in $19.9 \%$ of KRAS exon 2 WT tumors $(n=1911)$. KRAS exon 3 mutations were found in $4.3 \%$ of patients, KRAS exon 4 in $6.7 \%$, NRAS exon 2 in $3.8 \%$, NRAS exon 3 in $4.8 \%$, and NRAS exon 4 in $0.5 \%$ of patients. Moreover, the efficacy of anti-EGFR therapy was significantly inferior for tumors in the new RAS mutant subgroup compared to tumors in the all RAS WT subgroup regarding PFS ( $p=0.001)$, OS $(p=0.008)$, and $R R(p=0.001)$. There was no significant difference detected regarding PFS $(p=0.88)$, OS $(p=0.35)$, or RR $(p=0.32)$ when the new RAS mutant subgroup was compared to the KRAS exon 2 mutant subgroup. In sum, this meta-analysis also confirmed the previous findings that patients with new RAS mutations do not benefit from anti-EGFR therapy [77]. Another study was performed by Schirripa et al. (2015) who analyzed mutations in KRAS and NRAS (codon 12, 13, and 61 in both genes) in 786 mCRC patients. KRAS mutations were detected in 393 patients (50\%). Among these patients, 308 patients (78\%) had a mutation in codon 12, 70 patients (18\%) in codon 13, and 16 patients (4\%) in codon 61. NRAS mutations were detected in 47 out of 321 KRAS and BRAF WT (15\%) patients or in 6\% of the total study population. Mutations in NRAS codon 12, 13, and 61 were detected in $14(30 \%), 6(13 \%)$, and 27 (57\%) patients, respectively. A small subgroup of 8 NRASmutated patients received anti-EGFR therapy and was evaluated for response to treatment. Five of these patients were treated with cetuximab plus irinotecan, 2 patients received cetuximab monotherapy and 1 patient received panitumumab monotherapy. Seven of these patients did not respond to therapy and showed disease progression, while 1 patient showed initial disease stabilization. After eight weeks, this patient also experienced disease progression. This study confirms the negative predictive effect of NRAS mutations on anti-EGFR therapy [78].

\section{Prognostic value of new RAS mutations}

Next to the predictive value of new RAS mutations, there was also growing interest in their prognostic value. First, the prognostic role of new RAS mutations in patients receiving anti-EGFR therapy will be discussed, followed by the prognostic role of new RAS mutations in patients receiving other therapies. 
501 The survival results of the aforementioned studies that are summarized in table 3, suggest that new RAS mutations have a negative prognostic effect in $\mathrm{mCRC}$ patients treated with anti-EGFR therapy. Among the group of patients treated with anti-EGFR therapy in each study, an increased PFS and OS is seen in the RAS WT group compared to the new RAS-mutated group, pointing towards a negative prognostic value of RAS mutations in patients treated with anti-EGFR therapy $[20,48,62,63,67,69,72,74,76]$. However, Jonker et al. (2008) compared survival results in mCRC patients treated with cetuximab plus best supportive care or best supportive care alone. They reported that the mutation status of KRAS had no influence on survival among patients treated with supportive care alone [45]. In the aforementioned studies, cetuximab treatment was never compared to best supportive care. Therefore, it seems that no hard conclusions can be made on the negative prognostic effect of RAS mutations. However, it remains clear that RAS-mutated patients should not be treated with anti-EGFR therapy as they experience no survival benefit.

\subsection{Other therapies}

514 The role of new RAS mutations in CRC patients that were not treated with anti-EGFR therapy has also 515 been investigated. Some studies analyzed only the role of KRAS codon 61, while in other studies all new 516 RAS mutations were analyzed.

517 Richman et al. (2009) investigated whether KRAS mutations were associated with prognosis in advanced 518 CRC. Therefore, they assessed the mutation status of KRAS codon 12, 13 and 61 in patients participating 519 in the MRC FOCUS trial (Medical Research Council Fluorouracil, Oxaliplatin and Irinotecan: Use and 520 Sequencing). Patients were randomly assigned to different sequences of chemotherapy, including first521 line FU alone, FU/irinotecan, or FU/oxaliplatin. KRAS mutations were detected in $288(40.5 \%)$ and 23 522 (3.2\%) patients in exon 2 (codon 12/13) and codon 61, respectively. Although there was no difference in 523 PFS ( $\mathrm{p}=0.09)$, patients with KRAS-mutated tumors had significantly worse OS than KRAS WT patients $524(\mathrm{p}=0.008)$. The authors concluded that KRAS mutations are associated with poor prognosis in advanced 525 CRC [79].

526 Stremitzer et al. (2012) investigated the influence of the KRAS mutation status on recurrence-free 527 survival (RFS) and OS in patients with resectable colorectal cancer liver metastases receiving neo528 adjuvant chemotherapy including bevacizumab before liver resection. KRAS mutations were found in $52925 \%$ of these patients (15/60). Among these patients, 8,4 and 3 patients had a mutation in codon 12,13 , 530 and 61, respectively. When they compared the KRAS WT patients to KRAS-mutated patients, a significant 531 difference in median RFS (12.4 vs. 5.3 months, $p=0.037$ ) and median OS (not reached by time of analysis 
532 (median follow-up 37.5 months) vs. 31.8 months, $\mathrm{p}=0.011$ ) was found. The authors concluded that KRAS 533 mutations had a negative prognostic effect on RFS and OS [80].

534 Vauthey et al. (2013) studied the prognostic impact of the RAS (KRAS and NRAS) mutation status in 193 535 patients that had curative resection of colorectal cancer liver metastases after single-regimen 536 chemotherapy. RAS mutations were found in 34 patients (18\%). Among these patients, 29, 3, and 2 537 patients harbored mutations at codon 12, 61, and 13, respectively. A significant difference in 3-year 538 overall survival rate was seen between RAS WT patients and RAS-mutated patients ( $81 \%$ vs. $52.2 \%$, $539 \mathrm{p}=0.002)$. These results indicate that RAS mutation status is an independent predictor of OS after 540 resection of colorectal liver metastases. In addition, compared to RAS WT patients, RAS-mutated 541 patients had a significantly shorter 3 -year lung RFS rate $(34.6 \%$ vs. $59.3 \%, p<0.001)$, but there was no 542 significant difference in 3-year liver RFS rate (43.8\% vs. $50.2 \%, p=0.181)$ [81].

543 Yaeger et al. (2014) studied the effect of RAS mutations on OS in a cohort of $918 \mathrm{mCRC}$ patients. The 544 mutation status of KRAS and NRAS was assessed in codon 12,13,61, 117, and 146. RAS mutations were 545 found in 441 cases, including 394 KRAS exon 2-mutated cases, 19 KRAS exon 3-mutated cases, 10 KRAS 546 exon 4-mutated cases, 8 NRAS exon 2-mutated cases, and 10 NRAS exon 3-mutated cases. A worse OS 547 was associated with the occurrence of these mutations. Among RAS WT patients, the median OS was 81 548 months, while the median OS was only 47 months in RAS-mutated patients $(p<0.001)$ [82].

549 Mise et al. (2014) evaluated whether the mutation status of RAS has an impact on survival in 184 550 patients undergoing liver resection for colorectal liver metastases. They studied mutations in KRAS and 551 NRAS codon 12, 13, 61, and 146. RAS mutations were detected in 38 patients (21\%), 32 patients had 552 mutations in KRAS, 6 patients had mutations in NRAS. The authors found that the 5-year OS rate was 553 significantly higher in RAS WT patients compared to RAS-mutated patients $(61.6 \% \mathrm{vs.} 23.2 \%, \mathrm{p}<0.001)$ 554 [83].

555 In the aforementioned study of Schirripa et al. (2015) (see section 5.3 Cetuximab- or panitumumab556 based therapy) the prognostic role of KRAS and NRAS mutation status was studied in a cohort of mCRC 557 patients, most of them were not treated with anti-EGFR therapy. Compared to all RAS WT patients who 558 had a median OS of 42.7 months, a significantly shorter OS was seen in patients with mutations in NRAS 559 (25.6 months, $\mathrm{p}=0.0013)$ and $\operatorname{KRAS}(30.2$ months, $\mathrm{p}=0.0015)$. These results suggest a potential negative 560 prognostic role of RAS mutations in mCRC patients [78].

561 Most of these studies suggest a negative prognostic role of RAS mutations. However, looking at the 562 treatment arms that do not contain anti-EGFR therapy in table 3, survival results of both RAS-mutated 563 and RAS WT patients are comparable in some studies [67,69]. In sum, no conclusions can be drawn on 
this theme. It seems that the prognostic value of new RAS mutations is depending on the treatment that patients are receiving.

\section{Considerations regarding RAS evaluation}

Since RAS evaluation is affected by many factors, we will report the most important considerations regarding RAS mutation analysis in this part. First, the quality and origin of the starting material is important. DNA is usually isolated from formalin-fixed, paraffin-embedded (FFPE) material of the primary tumor. Therefore, the quality of DNA is often suboptimal due to chemical degradation of DNA in FFPE samples, cold ischemia, or delayed fixation [84, 85]. Ideally, fresh frozen tissue should be used, but unfortunately, frozen material is often lacking.

Next to the quality of DNA, variable handling before DNA extraction can also affect the results of the mutation analysis. Microdissection of the tumor tissue increases the purity of tumoral DNA but this technique is labor intensive and therefore not often performed. In the current FDA-approved assay, microdissection is only recommended for patients where less than $20 \%$ of the cells are cancerous. In addition, estimation of the percentage of tumoral cells in the specimen holds substantial interobserver variation $[86,87]$.

Another important consideration is whether a single biopsy of the primary tumor is sufficient for mutation analysis as intratumor heterogeneity has been shown [88]. A possible solution are liquid biopsies, consisting of circulating cell-free DNA and circulating tumor cells present in the blood of cancer patients. It has been reported that these liquid biopsies reflect the total systemic tumor burden [89] and it is possible to detect mutations in these liquid biopsies of patients with advanced cancer [90-95]. However, further research needs to be performed before liquid biopsies can be implemented in the clinic.

In addition, the used detection platforms may also affect the results of RAS mutation analysis. Sanger sequencing has been used for many years, but this technique has a sensitivity of only $20 \%[4,96]$. Currently, the only FDA-approved test for analyzing mutations in KRAS codon 12 or 13 uses the Scorpion Amplified Refractory Mutation System (ARMS) polymerase chain reaction methodology with a reported sensitivity of approximately $1 \%$ to $5 \%$. More sensitive technologies, such as digital PCR, BEAMing, and many next generation sequencing platforms reach a sensitivity up to $0.1 \%[1,87,97]$. There is a growing need for these sensitive platforms in order to serially monitor tumor burden and the emergence of acquired resistance mutations in liquid biopsies. However, it remains to be questioned which methodologies should be used for mutation analysis. The existing commercial KRAS mutation kits cannot be used for mutation analysis of KRAS exon 4 and NRAS exon 2, 3, and 4 . Adding these exons to existing 
kits will take a lot of time and money. Therefore, it is likely that targeted panels, sequenced through next generation sequencing will soon replace the traditional Sanger sequencing and allele-specific methods that are clinically used at this moment. In future, these panels can be quickly adapted when new negative predictive mutations are detected.

600 Another important consideration is the lower limit of detection of mutation that has a clinical relevance 601 in the treatment of patients with anti-EGFR therapy. In other words, how many mutant alleles have to be 602 present in order to predict unresponsiveness to anti-EGFR therapy? Laurent-Puig et al. (2015) used 603 picodroplet digital procedures to perform a mutation analysis on the tumor tissue of CRC patients. They 604 found an inverse correlation between the proportion of mutated DNA and the frequency of response to 605 anti-EGFR therapy. However, patients with less than $1 \%$ of mutant KRAS alleles did respond to anti-EGFR 606 therapy and had similar PFS and OS results as patients with wild-type KRAS tumors [4]. Therefore, it 607 seems to be needless to exclude these patients for anti-EGFR therapy. However, large prospective 608 studies are needed to perform further research on this theme and to translate these findings in clinical 609 settings. At this moment, the predictive value of low frequency RAS mutations remains unclear. It is 610 possible that these patients first respond to therapy and develop resistance after a few months of 611 treatment. The existence of acquired resistance has already been shown by Diaz et al. (2012) who 612 showed that $38 \%$ of patients that were initially classified as having wild-type tumors, developed 613 detectable KRAS mutations in serum during or after treatment [5]. Acquired resistance can be caused by 614 a few cancer cells harboring RAS mutations that expand during treatment, while wild-type cells are 615 dying. On the other hand, acquired resistance can also be caused by new RAS mutations that arise during 616 treatment $[5,37]$.

617 Finally, currently, there is no recommendation on the appropriate timing of RAS mutation determination, 618 except that the mutation analysis needs to be done before the start of anti-EGFR therapy. In the NCCN 619 Guidelines it is also recommended not to perform KRAS/NRAS genotyping at the early stage I, II or III 620 disease, as anti-EGFR agents are only used in the treatment of metastatic colorectal cancer [11]. We 621 suggest to perform RAS mutation analysis on fresh tumor tissue, preferably from metastatic origin, 622 obtained just before the start of anti-EGFR treatment. In this way, analyzing old tumor tissue of which 623 the tumor characteristics have possibly been changed over time due to chemotherapy or evolution of 624 the tumor is avoided and a real-time reflection of the metastasis is obtained.

\section{8. Discussion and conclusion}

626 Anti-EGFR therapy significantly improves the clinical outcomes of patients with mCRC. The main drawback associated with this therapy is the occurrence of resistance. Many patients are resistant to this 
628

629

630

631

632

633

634

635

636

637

638

639

640

641

642

643

644

645

646

647

648

649

650

651

652

653

654

655

656

657

658

659

therapy and almost all patients develop resistance within a few months after treatment start. In addition, anti-EGFR therapy is also associated with high costs and harmful side effects [3]. Despite the restriction of this therapy to patients that are WT for KRAS exon 2, up to $60 \%$ of these patients do not respond to cetuximab or panitumumab $[18,19]$. Improving patient selection might lead to better survival outcomes and quality of life for these patients.

In the last five years, a lot of research has been performed on the so-called new RAS mutations, as they might predict responsiveness to anti-EGFR therapy. The NCCN and EMA Guidelines have been updated recommending the restriction of anti-EGFR therapy to all RAS WT patients $[11,22,23]$. Recently, the FDA guidelines have also been changed reflecting the predictive role of all RAS mutations [47, 51, 52].

One aspect that impedes the study of the different RAS mutations is their low frequency. The majority of RAS mutations occur in codon $12(23.9 \%)$ and $13(3.6 \%)$ in exon 2 of KRAS, but the restriction of antiEGFR therapy to KRAS exon 2 WT mCRC patients has already been recommended since 2008. Less common mutations occur in codon 12 (2.1\%) of exon 2 and codon 61 (3.6\%) of exon 3 of NRAS and in codon 61 (1.4\%) of exon 3 and codon 146 (3.3\%) of exon 4 of KRAS [98]. However, regarding the aforementioned studies, new RAS mutations were detected in 15 to $26 \%$ of patients (Table 1), which accounts for a considerable number of mCRC patients. Here, we looked only at the studies in which the mutation status of KRAS exon 3 and 4 and NRAS exon 2, 3, and 4 were all analyzed in a KRAS exon 2 WT population, as the amount of new RAS-mutated patients in those populations are clinically important.

Regarding response to anti-EGFR therapy in different studies, it has been shown that the RR is lower in all RAS-mutated patients compared to all RAS WT patients (Table 2). Some studies reported no response in all analyzed RAS-mutated patients [62, 72] or in all NRAS-mutated patients [21]. Other studies reported sporadic cases with a mutation in $R A S$ that showed partial response [21, 48, 55, 73, 78].

The survival results in all studies generally showed a difference in PFS and OS in patients with new RAS mutations compared to all RAS WT patients that were treated with anti-EGFR therapy (Table 3). In the re-analysis of the PRIME trial, the addition of panitumumab to FOLFOX4 seemed to be detrimental for RAS-mutated patients [20]. This was also the case in the OPUS trial where cetuximab was added to FOLFOX4 [67]. Furthermore, in the PEAK and FIRE-3 trial, the addition of anti-EGFR therapy to chemotherapy in RAS-mutated patients generally resulted in worse outcome than the addition of bevacizumab to chemotherapy $[63,74]$.

The results of all these studies indicate that anti-EGFR therapy should be restricted to all RAS WT mCRC patients. Although the frequency of different mutations in the population is minor, the frequency of all RAS mutations together reaches $15 \%$ to $26 \%$ in a subgroup of patients that have no mutations in KRAS 
660

exon 2. Most of these patients showed no response to anti-EGFR therapy and had poor survival results. Although there was a very small subgroup of patients that showed response to treatment, none of these patients should be treated with anti-EGFR therapy in order to improve treatment possibilities in the vast majority of patients with new RAS mutations. This is a relatively large group of patients which are exposed to unnecessary toxicities and costs. If these patients can immediately be treated with another and possibly more effective therapy, the survival and quality of life of many mCRC patients might increase.

In conclusion, it is advised to perform a mutation analysis on KRAS and NRAS codons 12 and 13 (exon 2), 59 and 61 (exon 3), and 117 and 146 (exon 4) on the tissue of all mCRC patients before treatment with anti-EGFR therapy. The used mutation detection platform is of minor importance, as long as there is enough expertise and the methodology is sensitive enough to detect mutations at an allele frequency threshold of $\leq 5 \%$. We advise to work with this threshold until the predictive value of low frequency RAS mutations is clear. We believe that a lot of patients will benefit from the expanded mutation analysis, as patients with new RAS mutations will not be exposed to unnecessary toxicities and costs. However, it is clear that even with extended RAS testing, some patients will still not respond to anti-EGFR therapy. For those patients, further research is necessary in order to identify other biomarkers.

\section{Compliance with Ethical Standards:}

This work was supported by a grant of the National Cancer Plan, Belgium (grant: NKP 29_38). Nele Boeckx has no conflict of interest to declare. Prof. Dr. Marc Peeters has received grants from Amgen, Roche, Novartis, and Bayer and consulting fees and payment for lectures from Amgen, Bayer, Novartis, Sanofi, and Merck Serono. Prof. Dr. Guy Van Camp has no conflict of interest to declare. Prof. Dr. Patrick Pauwels has received fees from Merck for advisory board meetings and lectures. Dr. Ken Op de Beeck has no conflict of interest to declare. Dr. Vanessa Deschoolmeester has no conflict of interest to declare. 
Table 1. Overview of all new RAS mutations and their frequency detected in different studies

\begin{tabular}{|c|c|c|c|c|c|}
\hline \multirow[b]{2}{*}{ Study } & \multicolumn{3}{|c|}{ New RAS mutations analyzed } & \multirow[b]{2}{*}{ Mutation \% } & \multirow[b]{2}{*}{ Total \% } \\
\hline & Population & Gene, codon (or exon) & No. & & \\
\hline \multirow{2}{*}{$\begin{array}{l}\text { Loupakis et } \\
\text { al. [62] }\end{array}$} & \multirow{2}{*}{ KRAS exon 2 WT } & $K R A S, 61$ & 7 & 8 & \multirow{2}{*}{9} \\
\hline & & KRAS, 146 & 1 & 1 & \\
\hline \multirow{4}{*}{$\begin{array}{l}\text { De Roock } \\
\text { et al. [48] }\end{array}$} & \multirow{4}{*}{ Unselected } & KRAS, 59 & 1 & 0,001 & \multirow{3}{*}{ KRAS: 4,101 } \\
\hline & & KRAS, 61 & 16 & 2,1 & \\
\hline & & KRAS, 146 & 15 & 2 & \\
\hline & & NRAS, 12, 13, 61 & 17 & 2,6 & NRAS: 2,6 \\
\hline FIRE-3 [63] & KRAS exon 2 WT & $\begin{array}{c}\text { KRAS, 61, 146; } \\
\text { NRAS, 12, 13, 59, 61, 117, } 146\end{array}$ & 65 & 16 & 16 \\
\hline OPUS [67] & KRAS exon 2 WT & $K R A S$, exon 3,$4 ; N R A S$, exon $2,3,4$ & 31 & 26 & 26 \\
\hline $\begin{array}{l}\text { CRYSTAL } \\
\text { [70] }\end{array}$ & KRAS exon 2 WT & $\begin{array}{c}\text { KRAS, 59, 61, 117, 146; } \\
\text { NRAS, 12, 13, 59, 61, 117, } 146\end{array}$ & 63 & 15 & 15 \\
\hline \multirow{2}{*}{$\begin{array}{c}\text { Peeters et } \\
\text { al. [21] }\end{array}$} & \multirow{2}{*}{ Unselected } & $K R A S, 61$ & 7 & 2,5 & \multirow{2}{*}{7,5} \\
\hline & & NRAS, 12, 13, 61 & 14 & 5 & \\
\hline \multirow{5}{*}{ PRIME [20] } & \multirow{5}{*}{ KRAS exon 2 WT } & KRAS, 61 & 24 & 3,7 & \multirow{5}{*}{16,8} \\
\hline & & KRAS, 117, 146 & 36 & 5,6 & \\
\hline & & NRAS, 12, 13 & 22 & 3,4 & \\
\hline & & NRAS, 61 & 26 & 4,1 & \\
\hline & & NRAS, 117, 146 & 0 & 0 & \\
\hline \multirow{6}{*}{$\begin{array}{c}\text { André et } \\
\text { al. [72] }\end{array}$} & \multirow{6}{*}{ KRAS exon 2 WT } & KRAS, 59 & 1 & 1,7 & \multirow{6}{*}{15,1} \\
\hline & & KRAS, 61 & 3 & 5 & \\
\hline & & KRAS, 117, 146 & 0 & 0 & \\
\hline & & NRAS, 12 & 1 & 1,7 & \\
\hline & & NRAS, 13 & 1 & 1,7 & \\
\hline & & NRAS, 61 & 3 & 5 & \\
\hline \multirow{2}{*}{$\begin{array}{c}20020408 \\
{[73]}\end{array}$} & \multirow{2}{*}{ KRAS exon 2 WT } & KRAS exon 4 & 9 & 3,7 & \multirow{2}{*}{4,5} \\
\hline & & NRAS exon 4 & 2 & 0,8 & \\
\hline \multirow{5}{*}{ PEAK [74] } & \multirow{5}{*}{ KRAS exon 2 WT } & KRAS, 59, 61 & 9 & 4,1 & \multirow{5}{*}{23,1} \\
\hline & & KRAS, 117, 146 & 17 & 7,7 & \\
\hline & & NRAS, 12, 13 & 12 & 5,4 & \\
\hline & & NRAS, 59, 61 & 13 & 5,9 & \\
\hline & & NRAS, 117, 146 & 0 & 0 & \\
\hline $\begin{array}{l}20050181 \\
{[76]}\end{array}$ & KRAS exon 2 WT & $K R A S$, exon 3,$4 ; N R A S$, exon $2,3,4$ & 107 & 18 & 18 \\
\hline Molinari et & Unselected & KRAS, 60 & 1 & 1 & 4 \\
\hline al. [55] & Unselected & KRAS, 61 & 3 & 3 & 4 \\
\hline & & KRAS, 59, 61 & NA & 4,3 & \\
\hline & & KRAS, 117, 146 & NA & 6,7 & \\
\hline soricn et & KRAS exon 2 WT & NRAS, 12,13 & NA & 3,8 & 19,9 \\
\hline & & NRAS, 59, 61 & NA & 4,8 & \\
\hline & & NRAS, 117, 146 & NA & 0,5 & \\
\hline & & KRAS, 61 & 16 & 2 & \\
\hline Schirripa & Unselected & NRAS, 12 & 14 & 1,8 & 79 \\
\hline et al. [78] & Unselected & NRAS, 13 & 6 & 0,7 & 1,9 \\
\hline & & NRAS, 61 & 27 & 3,4 & \\
\hline
\end{tabular}


Table 2. Response rates of evaluable new RAS-mutated patients in different studies

\begin{tabular}{|c|c|c|c|c|c|c|c|}
\hline Study & $\frac{\text { RAS mutations analyzed }}{\text { Gene, codon (or exon) }}$ & $\begin{array}{c}\text { Treatment } \\
\text { (line of treatment) }\end{array}$ & $\frac{\text { RAS MT }}{\text { No. }}$ & $\frac{\text { RAS WT }}{\text { No. }}$ & $\frac{\text { RAS MT }}{\text { RR (\%) }}$ & $\frac{\text { RAS WT }}{\operatorname{RR}(\%)}$ & Remarks \\
\hline $\begin{array}{l}\text { Loupakis } \\
\text { et al. [62] }\end{array}$ & KRAS, 61, 146 & $\begin{array}{c}\text { cmab + irinotecan } \\
\text { (advanced lines) }\end{array}$ & 8 & 68 & 0 & 32,4 & $p=0,096$ \\
\hline \multirow{4}{*}{$\begin{array}{l}\text { De Roock } \\
\text { et al. [48] }\end{array}$} & $K R A S, 12,13,59,61,146$ & \multirow{4}{*}{$\begin{array}{c}\text { cmab + CT } \\
\text { (advanced lines) }\end{array}$} & 253 & 352 & 6,7 & $35,8^{*}$ & $p<0,0001$ \\
\hline & KRAS, 61 & & 13 & 352 & 0 & $35,8^{*}$ & \\
\hline & KRAS, 146 & & 11 & 352 & 18,2 & $35,8^{*}$ & $\mathrm{PR}$ in 2 KRAS $146 \mathrm{MT}$ patients \\
\hline & NRAS, 12, 13, 61 & & 13 & 289 & 7,7 & 38,1 & $\mathrm{p}=0,013 ; \mathrm{R}$ in $1 \mathrm{NRAS} \mathrm{MT}$ patient \\
\hline \multirow{2}{*}{$\begin{array}{l}\text { FIRE-3 } \\
{[63]}\end{array}$} & \multirow{2}{*}{ KRAS, 61, 146; NRAS, 12, 13, 61, 117, 146} & FOLFIRI + bmab & 31 & 171 & 58 & 60 & \\
\hline & & $\begin{array}{l}\text { FOLFIRI + cmab } \\
\text { (first-line) }\end{array}$ & 34 & 171 & 38 & 65 & \\
\hline \multirow{2}{*}{$\begin{array}{l}\text { OPUS } \\
{[67]}\end{array}$} & \multirow{2}{*}{ KRAS, exon 3,$4 ; N R A S$, exon $2,3,4$} & FOLFOX4 & 16 & 49 & 43,8 & 28,6 & \\
\hline & & $\begin{array}{l}\text { FOLFOX4 + cmab } \\
\text { (first-line) }\end{array}$ & 15 & 38 & 53,3 & 57,9 & \\
\hline \multirow{2}{*}{$\begin{array}{l}\text { CRYSTAL } \\
\text { [70] }\end{array}$} & \multirow{2}{*}{ KRAS, 59, 61, 117, 146; NRAS, 12, 13, 59, 61, 117, 146} & FOLFIRI & 31 & 189 & 35,5 & 38,6 & \\
\hline & & $\begin{array}{l}\text { FOLFIRI + cmab } \\
\text { (first-line) }\end{array}$ & 32 & 178 & 34,4 & 66,3 & \\
\hline \multirow{2}{*}{$\begin{array}{l}\text { Peeters } \\
\text { et al. [21] }\end{array}$} & KRAS, 12, 13, 61 & \multirow{2}{*}{$\begin{array}{c}\text { pmab MT } \\
\text { (advanced lines) }\end{array}$} & 109 & 126 & 1 & 17 & $\mathrm{PR}$ in $1 / 6$ codon $61 \mathrm{MT}$ patients \\
\hline & NRAS, 12, 13, 61 & & 9 & 126 & 0 & 17 & \\
\hline $\begin{array}{l}\text { André et } \\
\text { al. [72] }\end{array}$ & $K R A S, 12,59,61 ;$ NRAS, $12,13,61$ & $\begin{array}{l}\text { irinotecan + pmab } \\
\text { (third-line) }\end{array}$ & 15 & 45 & 0 & 46,3 & \\
\hline $\begin{array}{c}20020408 \\
{[73]}\end{array}$ & $K R A S$ and $N R A S$, exon $2,3,4$ & $\begin{array}{c}\text { pmab } \\
\text { (advanced lines) }\end{array}$ & 95 & 72 & 1 & 15 & PR in 1 KRAS exon $4 \mathrm{MT}$ patient \\
\hline \multirow{3}{*}{$\begin{array}{l}\text { Molinari } \\
\text { et al. [55] }\end{array}$} & KRAS, 12,13 & \multirow{3}{*}{$\begin{array}{l}\text { cmab- or pmab-based } \\
\text { regimen } \\
\text { (first- or advances lines) }\end{array}$} & 43 & 64 & 4,7 & 28,1 & PR in 2 KRAS G13D MT patients \\
\hline & KRAS, 60 & & 1 & 64 & 100 & 28,1 & \\
\hline & KRAS, 61 & & 3 & 64 & 0 & 28,1 & also other mutations present \\
\hline $\begin{array}{l}\text { Schirripa } \\
\text { et al. [78] }\end{array}$ & NRAS, 12, 13, 61 & $\begin{array}{l}\text { cmab + irinotecan, or } \\
\text { cmab MT, or pmab MT } \\
\text { (advanced lines) }\end{array}$ & 8 & NA & 12,5 & NA & PR in 1 patient \\
\hline
\end{tabular}

WT: wild-type; MT: mutated; NA: data not available; cmab: cetuximab; pmab: panitumumab; bmab: bevacizumab; MT: monotherapy; RR: response rate; PR: partial response; R: response; CT:

chemotherapy; No.: Number of patients harboring the specified mutation(s); ${ }^{*}$ compared to KRAS exon 2 WT patients instead of all RAS WT patients 
Table 3. Survival outcomes of new RAS-mutated patients and all RAS WT patients in different studies

\begin{tabular}{|c|c|c|c|c|c|c|c|c|}
\hline \multirow[b]{2}{*}{ Study } & \multicolumn{2}{|l|}{ new RAS mutations analyzed } & New RAS MT & \multirow{2}{*}{$\frac{\text { RAS WT }}{\text { No. }}$} & \multicolumn{2}{|c|}{ New RAS MT } & \multicolumn{2}{|c|}{ all RAS WT } \\
\hline & Gene, codon (or exon) & $\begin{array}{c}\text { Treatment } \\
\text { (line of treatment) }\end{array}$ & No. & & $\begin{array}{c}\text { PFS } \\
\text { (months) }\end{array}$ & $\begin{array}{c}\text { OS } \\
\text { (months) }\end{array}$ & $\begin{array}{c}\text { PFS } \\
\text { (months) }\end{array}$ & $\begin{array}{c}\text { OS } \\
\text { (months) }\end{array}$ \\
\hline \multirow{2}{*}{$\begin{array}{l}\text { Loupakis } \\
\text { et al. [62] }\end{array}$} & \multirow[t]{2}{*}{ KRAS, 61, 146} & Irinotecan + cmab & 8 & 68 & 3,8 & 9,7 & 5,1 & 14,7 \\
\hline & & (advanced lines) & \multicolumn{6}{|c|}{$p(P F S)=0,028 ; p(O S)=0,390$} \\
\hline \multirow{4}{*}{$\begin{array}{l}\text { De Roock } \\
\text { et al. [48] }\end{array}$} & \multirow[t]{2}{*}{$K R A S, 12,13,59,61,146$} & $\mathrm{CT}+\mathrm{cmab}$ & 253 & 352 & 3 & 8 & 6 & 12,5 \\
\hline & & & \multicolumn{6}{|c|}{$p(P F S$ and $O S)<0,0001$} \\
\hline & \multirow{2}{*}{ NRAS, 12, 13, 61} & $\mathrm{CT}+\mathrm{cmab}$ & 13 & 289 & 3,5 & 9,5 & 6,5 & 12,5 \\
\hline & & (advanced lines) & \multicolumn{6}{|c|}{$p(P F S)=0,055 ; p(O S)=0,051$} \\
\hline \multirow{3}{*}{$\begin{array}{c}\text { FIRE-3 } \\
\text { [63] }\end{array}$} & \multirow{3}{*}{ KRAS, 61, 146; NRAS, 12, 13, 61, 117, 146} & FOLFIRI + bmab & 31 & 171 & 12,2 & 20,6 & 10,2 & 25,6 \\
\hline & & FOLFIRI + cmab & 34 & 171 & 6,1 & 16,4 & 10,4 & 33,1 \\
\hline & & (first-line) & & & $p=0,004$ & $p=0,57$ & $p=0,54$ & $p=0,011$ \\
\hline \multirow{3}{*}{$\begin{array}{c}\text { OPUS } \\
\text { [67] }\end{array}$} & \multirow{3}{*}{$K R A S$, exon 3, 4; NRAS, exon 2, 3, 4} & FOLFOX4 & 16 & 49 & 7,4 & 17,8 & 5,8 & 17,8 \\
\hline & & FOLFOX4 + cmab & 15 & 38 & 7,5 & 18,4 & 12 & 19,8 \\
\hline & & (first-line) & & & $p=0,60$ & $p=0,86$ & $p=0,062$ & $p=0,80$ \\
\hline \multirow{3}{*}{$\begin{array}{c}\text { CRYSTAL } \\
\text { [70] }\end{array}$} & \multirow{3}{*}{ KRAS, 59, 61, 117, 146; NRAS, 12, 13, 59, 61, 117, 146} & FOLFIRI & 31 & 189 & 6,9 & 20,7 & 8,4 & 20,2 \\
\hline & & FOLFIRI + cmab & 32 & 178 & 7,2 & 18,2 & 11,4 & 28,4 \\
\hline & & (first-line) & & & $p=0,56$ & $p=0,5$ & $p=0,0002$ & $p=0,0024$ \\
\hline \multirow{3}{*}{$\begin{array}{l}\text { PRIME } \\
\text { [20] }\end{array}$} & \multirow{3}{*}{ KRAS, 61, 117, 146; NRAS, 12, 13, 61} & FOLFOX4 & 57 & 253 & 8 & 17,8 & 7,9 & 20,2 \\
\hline & & FOLFOX4 + pmab & 51 & 259 & 7,3 & 17,1 & 10,1 & 26 \\
\hline & & (first-line) & & & $p=0,33$ & $p=0,12$ & $p=0,004$ & $p=0,04$ \\
\hline \multirow{2}{*}{$\begin{array}{l}\text { André et } \\
\text { al. [72] }\end{array}$} & \multirow[t]{2}{*}{ KRAS, 12, 59, 61; NRAS, 12, 13, 61} & irinotecan + pmab & 15 & 45 & 1,9 & 4,6 & 8,7 & 15,8 \\
\hline & & (third-line) & & & $N A$ & $N A$ & $N A$ & $N A$ \\
\hline \multirow{3}{*}{ PEAK [74] } & \multirow{3}{*}{ KRAS, exon 3,$4 ; N R A S$, exon 2,3} & mFOLFOX6 + bmab & 27 & 82 & 8,9 & 16,6 & 9,5 & 28,9 \\
\hline & & mFOLFOX6 + pmab & 24 & 88 & 7,8 & 27 & 13 & 41,3 \\
\hline & & (first-line) & & & $p=0,318$ & $p=0,020$ & $p=0,029$ & $p=0,058$ \\
\hline \multirow{3}{*}{$\begin{array}{c}20050181 \\
{[76]}\end{array}$} & \multirow{3}{*}{ KRAS, exon 3, 4; NRAS, exon 2, 3, 4} & FOLFIRI & 294 & 213 & $* 4,0$ & $* 11,1$ & 4,6 & 13,9 \\
\hline & & FOLFIRI + pmab & 299 & 208 & $* 4,8$ & $* 11,8$ & 6,4 & 16,2 \\
\hline & & (second-line) & & & $p=0,14$ & $p=0,34$ & $p=0,007$ & $p=0,08$ \\
\hline
\end{tabular}

NA: data not available; WT: wild-type; MT: mutated; PFS: progression-free survival; OS: overall survival; cmab: cetuximab; pmab: panitumumab, bmab: bevacuzimab; CT: chemotherapy; No.: Number of patients harboring the specified mutation(s); *: all RAS-mutated patients instead of only new-RAS mutated patients 


\section{References}

1. Leary RJ, Sausen M, Kinde I, Papadopoulos N, Carpten JD, Craig D et al. Detection of chromosomal alterations in the circulation of cancer patients with whole-genome sequencing. Science translational medicine. 2012;4(162):162ra54. doi:10.1126/scitranslmed.3004742.

2. Dressman D, Yan H, Traverso G, Kinzler KW, Vogelstein B. Transforming single DNA molecules into fluorescent magnetic particles for detection and enumeration of genetic variations. Proceedings of the National Academy of Sciences of the United States of America. 2003;100(15):8817-22. doi:10.1073/pnas.1133470100.

3. Deschoolmeester V, Baay M, Specenier P, Lardon F, Vermorken JB. A review of the most promising biomarkers in colorectal cancer: one step closer to targeted therapy. The oncologist. 2010;15(7):699731. doi:10.1634/theoncologist.2010-0025.

4. Laurent-Puig P, Pekin D, Normand C, Kotsopoulos SK, Nizard P, Perez-Toralla K et al. Clinical relevance of KRAS-mutated subclones detected with picodroplet digital PCR in advanced colorectal cancer treated with anti-EGFR therapy. Clinical cancer research : an official journal of the American Association for Cancer Research. 2015;21(5):1087-97. doi:10.1158/1078-0432.CCR-14-0983.

5. Diaz LA, Jr., Williams RT, Wu J, Kinde I, Hecht JR, Berlin J et al. The molecular evolution of acquired resistance to targeted EGFR blockade in colorectal cancers. Nature. 2012;486(7404):537-40. doi:10.1038/nature11219.

6. Baselga J. Why the epidermal growth factor receptor? The rationale for cancer therapy. The oncologist. 2002;7 Suppl 4:2-8.

7. Peeters M, Price TJ, Cervantes A, Sobrero AF, Ducreux M, Hotko Y et al. Randomized phase III study of panitumumab with fluorouracil, leucovorin, and irinotecan (FOLFIRI) compared with FOLFIRI alone as second-line treatment in patients with metastatic colorectal cancer. Journal of clinical oncology : official journal of the American Society of Clinical Oncology. 2010;28(31):4706-13. doi:10.1200/JCO.2009.27.6055.

8. Lievre A, Bachet JB, Le Corre D, Boige V, Landi B, Emile JF et al. KRAS mutation status is predictive of response to cetuximab therapy in colorectal cancer. Cancer research. 2006;66(8):3992-5. doi:10.1158/0008-5472.CAN-06-0191.

9. Karapetis CS, Khambata-Ford S, Jonker DJ, O'Callaghan CJ, Tu D, Tebbutt NC et al. K-ras mutations and benefit from cetuximab in advanced colorectal cancer. The New England journal of medicine. 2008;359(17):1757-65. doi:10.1056/NEJMoa0804385.

10. Amado RG, Wolf M, Peeters M, Van Cutsem E, Siena S, Freeman DJ et al. Wild-type KRAS is required for panitumumab efficacy in patients with metastatic colorectal cancer. Journal of clinical oncology : official journal of the American Society of Clinical Oncology. 2008;26(10):1626-34. doi:10.1200/JCO.2007.14.7116.

11. National Comprehensive Cancer Network. Clinical Practice Guidelines in Oncology (NCCN Guidelines). Colon Cancer. NCCN.org.

12. Cunningham $D$, Humblet $Y$, Siena $S$, Khayat $D$, Bleiberg $H$, Santoro $A$ et al. Cetuximab monotherapy and cetuximab plus irinotecan in irinotecan-refractory metastatic colorectal cancer. The New England journal of medicine. 2004;351(4):337-45. doi:10.1056/NEJMoa033025.

13. Van Cutsem E, Peeters M, Siena S, Humblet $Y$, Hendlisz A, Neyns B et al. Open-label phase III trial of panitumumab plus best supportive care compared with best supportive care alone in patients with chemotherapy-refractory metastatic colorectal cancer. Journal of clinical oncology : official journal of the American Society of Clinical Oncology. 2007;25(13):1658-64. doi:10.1200/JCO.2006.08.1620.

14. Saltz LB, Meropol NJ, Loehrer PJ, Sr., Needle MN, Kopit J, Mayer RJ. Phase II trial of cetuximab in patients with refractory colorectal cancer that expresses the epidermal growth factor receptor. Journal 
of clinical oncology : official journal of the American Society of Clinical Oncology. 2004;22(7):1201-8. doi:10.1200/JCO.2004.10.182.

15. Custodio A, Feliu J. Prognostic and predictive biomarkers for epidermal growth factor receptortargeted therapy in colorectal cancer: beyond KRAS mutations. Critical reviews in oncology/hematology. 2013;85(1):45-81. doi:10.1016/j.critrevonc.2012.05.001.

16. Peeters $\mathrm{M}$, Cohn $\mathrm{A}$, Kohne $\mathrm{CH}$, Douillard JY. Panitumumab in combination with cytotoxic chemotherapy for the treatment of metastatic colorectal carcinoma. Clinical colorectal cancer. 2012;11(1):14-23. doi:10.1016/j.clcc.2011.06.010.

17. Benvenuti S, Sartore-Bianchi A, Di Nicolantonio F, Zanon C, Moroni M, Veronese S et al. Oncogenic activation of the RAS/RAF signaling pathway impairs the response of metastatic colorectal cancers to anti-epidermal growth factor receptor antibody therapies. Cancer research. 2007;67(6):2643-8. doi:10.1158/0008-5472.CAN-06-4158.

18. Allegra CJ, Jessup JM, Somerfield MR, Hamilton SR, Hammond EH, Hayes DF et al. American Society of Clinical Oncology provisional clinical opinion: testing for KRAS gene mutations in patients with metastatic colorectal carcinoma to predict response to anti-epidermal growth factor receptor monoclonal antibody therapy. Journal of clinical oncology : official journal of the American Society of Clinical Oncology. 2009;27(12):2091-6. doi:10.1200/JCO.2009.21.9170.

19. Fakih M, Wong R. Efficacy of the monoclonal antibody EGFR inhibitors for the treatment of metastatic colorectal cancer. Current oncology. 2010;17 Suppl 1:S3-17.

20. Douillard JY, Oliner KS, Siena S, Tabernero J, Burkes R, Barugel M et al. Panitumumab-FOLFOX4 treatment and RAS mutations in colorectal cancer. The New England journal of medicine. 2013;369(11):1023-34. doi:10.1056/NEJMoa1305275.

21. Peeters M, Oliner KS, Parker A, Siena S, Van Cutsem E, Huang J et al. Massively parallel tumor multigene sequencing to evaluate response to panitumumab in a randomized phase III study of metastatic colorectal cancer. Clinical cancer research : an official journal of the American Association for Cancer Research. 2013;19(7):1902-12. doi:10.1158/1078-0432.CCR-12-1913.

22. European Medicines Agency. Erbitux. Available at: http://www.ema.europa.eu/ema/ index.jsp?curl=pages/medicines/human/medicines/000558/human_med_000769.jsp\&mid=WC0B01ACO 58001D124. Accessed December 29, 2014.

23. European Medicines Agency. Vectibix. Available at: http://www.ema.europa.eu/ema/ index.jsp?curl=pages/medicines/human/medicines/000741/human_med_001128.jsp\&mid=WC0B01AC0 58001D124. Accessed December 29, 2014.

24. Yarden Y. The EGFR family and its ligands in human cancer. signalling mechanisms and therapeutic opportunities. European journal of cancer. 2001;37 Suppl 4:S3-8.

25. Mahipal A, Kothari N, Gupta S. Epidermal growth factor receptor inhibitors: coming of age. Cancer control : journal of the Moffitt Cancer Center. 2014;21(1):74-9.

26. Goldstein NS, Armin M. Epidermal growth factor receptor immunohistochemical reactivity in patients with American Joint Committee on Cancer Stage IV colon adenocarcinoma: implications for a standardized scoring system. Cancer. 2001;92(5):1331-46.

27. Campanella C, Mottolese M, Cianciulli A, Torsello A, Merola R, Sperduti I et al. Epidermal growth factor receptor gene copy number in 101 advanced colorectal cancer patients treated with chemotherapy plus cetuximab. Journal of translational medicine. 2010;8:36. doi:10.1186/1479-5876-836.

28. Holbro T, Hynes NE. ErbB receptors: directing key signaling networks throughout life. Annual review of pharmacology and toxicology. 2004;44:195-217. doi:10.1146/annurev.pharmtox.44.101802.121440.

29. Ashraf N, Kothari N, Kim R. Predictive biomarkers for anti-epidermal growth factor receptor therapy: beyond KRAS testing. Journal of the National Comprehensive Cancer Network : JNCCN. 2014;12(10):1433-42. 
30. Matallanas D, Birtwistle M, Romano D, Zebisch A, Rauch J, von Kriegsheim A et al. Raf family kinases: old dogs have learned new tricks. Genes \& cancer. 2011;2(3):232-60. doi:10.1177/1947601911407323.

31. Charette N, Vandeputte C, Starkel P. Ras in digestive oncology: from molecular biology to clinical implications. Current opinion in oncology. 2014;26(4):454-61. doi:10.1097/CCO.0000000000000088.

32. Pylayeva-Gupta Y, Grabocka E, Bar-Sagi D. RAS oncogenes: weaving a tumorigenic web. Nature reviews Cancer. 2011;11(11):761-74. doi:10.1038/nrc3106.

33. Grewal T, Koese M, Tebar F, Enrich C. Differential Regulation of RasGAPs in Cancer. Genes \& cancer. 2011;2(3):288-97. doi:10.1177/1947601911407330.

34. Goel A, Arnold CN, Niedzwiecki D, Carethers JM, Dowell JM, Wasserman L et al. Frequent inactivation of PTEN by promoter hypermethylation in microsatellite instability-high sporadic colorectal cancers. Cancer research. 2004;64(9):3014-21.

35. Di Cristofano A, Pandolfi PP. The multiple roles of PTEN in tumor suppression. Cell. 2000;100(4):38790.

36. Sobrero AF, Maurel J, Fehrenbacher L, Scheithauer W, Abubakr YA, Lutz MP et al. EPIC: phase III trial of cetuximab plus irinotecan after fluoropyrimidine and oxaliplatin failure in patients with metastatic colorectal cancer. Journal of clinical oncology : official journal of the American Society of Clinical Oncology. 2008;26(14):2311-9. doi:10.1200/JCO.2007.13.1193.

37. Misale S, Di Nicolantonio F, Sartore-Bianchi A, Siena S, Bardelli A. Resistance to anti-EGFR therapy in colorectal cancer: from heterogeneity to convergent evolution. Cancer discovery. 2014;4(11):1269-80. doi:10.1158/2159-8290.CD-14-0462.

38. Van Cutsem E, Kohne CH, Hitre E, Zaluski J, Chang Chien CR, Makhson A et al. Cetuximab and chemotherapy as initial treatment for metastatic colorectal cancer. The New England journal of medicine. 2009;360(14):1408-17. doi:10.1056/NEJMoa0805019.

39. De Roock W, Piessevaux H, De Schutter J, Janssens M, De Hertogh G, Personeni N et al. KRAS wildtype state predicts survival and is associated to early radiological response in metastatic colorectal cancer treated with cetuximab. Annals of oncology : official journal of the European Society for Medical Oncology / ESMO. 2008;19(3):508-15. doi:10.1093/annonc/mdm496.

40. Douillard JY, Siena S, Cassidy J, Tabernero J, Burkes R, Barugel M et al. Randomized, phase III trial of panitumumab with infusional fluorouracil, leucovorin, and oxaliplatin (FOLFOX4) versus FOLFOX4 alone as first-line treatment in patients with previously untreated metastatic colorectal cancer: the PRIME study. Journal of clinical oncology : official journal of the American Society of Clinical Oncology. 2010;28(31):4697-705. doi:10.1200/JCO.2009.27.4860.

41. Peeters M, Douillard JY, Van Cutsem E, Siena S, Zhang K, Williams R et al. Mutant KRAS codon 12 and 13 alleles in patients with metastatic colorectal cancer: assessment as prognostic and predictive biomarkers of response to panitumumab. Journal of clinical oncology : official journal of the American Society of Clinical Oncology. 2013;31(6):759-65. doi:10.1200/JCO.2012.45.1492.

42. Lievre A, Bachet JB, Boige V, Cayre A, Le Corre D, Buc E et al. KRAS mutations as an independent prognostic factor in patients with advanced colorectal cancer treated with cetuximab. Journal of clinical oncology : official journal of the American Society of Clinical Oncology. 2008;26(3):374-9. doi:10.1200/JCO.2007.12.5906.

43. Di Fiore F, Blanchard F, Charbonnier F, Le Pessot F, Lamy A, Galais MP et al. Clinical relevance of KRAS mutation detection in metastatic colorectal cancer treated by Cetuximab plus chemotherapy. British journal of cancer. 2007;96(8):1166-9. doi:10.1038/sj.bjc.6603685.

44. Maughan TS, Adams RA, Smith CG, Meade AM, Seymour MT, Wilson RH et al. Addition of cetuximab to oxaliplatin-based first-line combination chemotherapy for treatment of advanced colorectal cancer: results of the randomised phase 3 MRC COIN trial. Lancet. 2011;377(9783):2103-14. doi:10.1016/S01406736(11)60613-2. 
45. Jonker DJ, O'Callaghan CJ, Karapetis CS, Zalcberg JR, Tu D, Au HJ et al. Cetuximab for the treatment of colorectal cancer. The New England journal of medicine. 2007;357(20):2040-8. doi:10.1056/NEJMoa071834.

46. Deschoolmeester V, Boeckx C, Baay M, Weyler J, Wuyts W, Van Marck E et al. KRAS mutation detection and prognostic potential in sporadic colorectal cancer using high-resolution melting analysis. British journal of cancer. 2010;103(10):1627-36. doi:10.1038/sj.bjc.6605959.

47. Cetuximab (Erbitux) and Panitumumab (Vectibix). U.S. Food and Drug Administration Web Site. Available at: http://www.fda.gov/AboutFDA/CentersOffices/OfficeofMedicalProductsandTobacco/CDER/ ucm172905. Accessed December 29, 2014.

48. De Roock W, Claes B, Bernasconi D, De Schutter J, Biesmans B, Fountzilas G et al. Effects of KRAS, BRAF, NRAS, and PIK3CA mutations on the efficacy of cetuximab plus chemotherapy in chemotherapyrefractory metastatic colorectal cancer: a retrospective consortium analysis. The Lancet Oncology. 2010;11(8):753-62. doi:10.1016/S1470-2045(10)70130-3.

49. De Roock W, De Vriendt V, Normanno N, Ciardiello F, Tejpar S. KRAS, BRAF, PIK3CA, and PTEN mutations: implications for targeted therapies in metastatic colorectal cancer. The Lancet Oncology. 2011;12(6):594-603. doi:10.1016/S1470-2045(10)70209-6.

50. Laurent-Puig P, Cayre A, Manceau G, Buc E, Bachet JB, Lecomte T et al. Analysis of PTEN, BRAF, and EGFR status in determining benefit from cetuximab therapy in wild-type KRAS metastatic colon cancer. Journal of clinical oncology : official journal of the American Society of Clinical Oncology. 2009;27(35):5924-30. doi:10.1200/JCO.2008.21.6796.

51. U.S. Food and Drug Administration. VECTIBIX. Available at: http://www.accessdata.fda.gov/scripts/ cder/drugsatfda/index.cfm?fuseaction=Search.DrugDetails. Accessed December 29, 2014.

52. U.S. Food and Drug Administration. ERBITUX. Available at: http://www.accessdata.fda.gov/scripts/ cder/drugsatfda/index.cfm?fuseaction=Search.DrugDetails. Accessed December 29, 2014.

53. De Roock W, Jonker DJ, Di Nicolantonio F, Sartore-Bianchi A, Tu D, Siena S et al. Association of KRAS p.G13D mutation with outcome in patients with chemotherapy-refractory metastatic colorectal cancer treated with cetuximab. Jama. 2010;304(16):1812-20. doi:10.1001/jama.2010.1535.

54. Tejpar S, Celik I, Schlichting M, Sartorius U, Bokemeyer C, Van Cutsem E. Association of KRAS G13D tumor mutations with outcome in patients with metastatic colorectal cancer treated with first-line chemotherapy with or without cetuximab. Journal of clinical oncology : official journal of the American Society of Clinical Oncology. 2012;30(29):3570-7. doi:10.1200/JCO.2012.42.2592.

55. Molinari F, Felicioni L, Buscarino M, De Dosso S, Buttitta F, Malatesta S et al. Increased detection sensitivity for KRAS mutations enhances the prediction of anti-EGFR monoclonal antibody resistance in metastatic colorectal cancer. Clinical cancer research : an official journal of the American Association for Cancer Research. 2011;17(14):4901-14. doi:10.1158/1078-0432.CCR-10-3137.

56. Guerrero S, Casanova I, Farre L, Mazo A, Capella G, Mangues R. K-ras codon 12 mutation induces higher level of resistance to apoptosis and predisposition to anchorage-independent growth than codon 13 mutation or proto-oncogene overexpression. Cancer research. 2000;60(23):6750-6.

57. Guerrero S, Figueras A, Casanova I, Farre L, Lloveras B, Capella G et al. Codon 12 and codon 13 mutations at the K-ras gene induce different soft tissue sarcoma types in nude mice. FASEB journal : official publication of the Federation of American Societies for Experimental Biology. 2002;16(12):16424. doi:10.1096/fj.02-0050fje.

58. Smith G, Bounds R, Wolf H, Steele RJ, Carey FA, Wolf CR. Activating K-Ras mutations outwith 'hotspot' codons in sporadic colorectal tumours - implications for personalised cancer medicine. British journal of cancer. 2010;102(4):693-703. doi:10.1038/sj.bjc.6605534.

59. Alamo P, Gallardo A, Di Nicolantonio F, Pavon MA, Casanova I, Trias M et al. Higher metastatic efficiency of KRas G12V than KRas G13D in a colorectal cancer model. FASEB journal : official publication of the Federation of American Societies for Experimental Biology. 2014. doi:10.1096/fj.14-262303. 
60. Schirripa M, Lonardi S, Cremolini C, Loupakis F, Salvatore L, Bergamo F et al. Phase II study of singleagent cetuximab in KRAS G13D mutant metastatic colorectal cancer (mCRC). Journal of clinical oncology : official journal of the American Society of Clinical Oncology. 2014;32:5s (suppl; abstr 3524).

61. Gajate P, Sastre J, Bando I, Alonso T, Cillero L, Sanz J et al. Influence of KRAS p.G13D mutation in patients with metastatic colorectal cancer treated with cetuximab. Clinical colorectal cancer. 2012;11(4):291-6. doi:10.1016/j.clcc.2012.02.003.

62. Loupakis F, Ruzzo A, Cremolini C, Vincenzi B, Salvatore L, Santini D et al. KRAS codon 61, 146 and BRAF mutations predict resistance to cetuximab plus irinotecan in KRAS codon 12 and 13 wild-type metastatic colorectal cancer. British journal of cancer. 2009;101(4):715-21. doi:10.1038/sj.bjc.6605177.

63. Heinemann V, von Weikersthal LF, Decker T, Kiani A, Vehling-Kaiser U, Al-Batran SE et al. FOLFIRI plus cetuximab versus FOLFIRI plus bevacizumab as first-line treatment for patients with metastatic colorectal cancer (FIRE-3): a randomised, open-label, phase 3 trial. The Lancet Oncology. 2014;15(10):1065-75. doi:10.1016/S1470-2045(14)70330-4.

64. Ciardiello F, Bianco R, Caputo R, Caputo R, Damiano V, Troiani T et al. Antitumor activity of ZD6474, a vascular endothelial growth factor receptor tyrosine kinase inhibitor, in human cancer cells with acquired resistance to antiepidermal growth factor receptor therapy. Clinical cancer research : an official journal of the American Association for Cancer Research. 2004;10(2):784-93.

65. Viloria-Petit A, Crombet T, Jothy S, Hicklin D, Bohlen P, Schlaeppi JM et al. Acquired resistance to the antitumor effect of epidermal growth factor receptor-blocking antibodies in vivo: a role for altered tumor angiogenesis. Cancer research. 2001;61(13):5090-101.

66. Bokemeyer C, Bondarenko I, Hartmann JT, de Braud F, Schuch G, Zubel A et al. Efficacy according to biomarker status of cetuximab plus FOLFOX-4 as first-line treatment for metastatic colorectal cancer: the OPUS study. Annals of oncology : official journal of the European Society for Medical Oncology / ESMO. 2011;22(7):1535-46. doi:10.1093/annonc/mdq632.

67. Bokemeyer C, Kohne CH, Ciardiello F, Lenz H, Heinemann V, Klinkhardt U et al. Treatment outcome according to tumor RAS mutation status in OPUS study patients with metastatic colorectal cancer (mCRC) randomized to FOLFOX4 with/without cetuximab. Journal of clinical oncology : official journal of the American Society of Clinical Oncology. 2014;32:5s, (suppl; abstr 3505).

68. Van Cutsem E, Kohne CH, Lang I, Folprecht G, Nowacki MP, Cascinu S et al. Cetuximab plus irinotecan, fluorouracil, and leucovorin as first-line treatment for metastatic colorectal cancer: updated analysis of overall survival according to tumor KRAS and BRAF mutation status. Journal of clinical oncology : official journal of the American Society of Clinical Oncology. 2011;29(15):2011-9. doi:10.1200/JCO.2010.33.5091.

69. Ciardiello F, Lenz $\mathrm{H}$, Kohne $\mathrm{CH}$, Heinemann V, Tejpar S, Esser R et al. Effect of KRAS nad NRAS mutational status on first-line treatment with FOLFIRI plux cetuximab in patients with metastatic colorectal cancer $(\mathrm{mCRC})$ : New results from the CRYSTAL trial. Journal of clinical oncology : official journal of the American Society of Clinical Oncology. 2014;32 (suppl; abstr LBA443).

70. Van Cutsem E, Lenz HJ, Kohne CH, Heinemann V, Tejpar S, Melezinek I et al. Fluorouracil, Leucovorin, and Irinotecan Plus Cetuximab Treatment and RAS Mutations in Colorectal Cancer. Journal of clinical oncology : official journal of the American Society of Clinical Oncology. 2015. doi:10.1200/JCO.2014.59.4812.

71. Douillard JY, Siena S, Cassidy J, Tabernero J, Burkes R, Barugel M et al. Final results from PRIME: randomized phase III study of panitumumab with FOLFOX4 for first-line treatment of metastatic colorectal cancer. Annals of oncology : official journal of the European Society for Medical Oncology / ESMO. 2014;25(7):1346-55. doi:10.1093/annonc/mdu141.

72. Andre T, Blons H, Mabro M, Chibaudel B, Bachet JB, Tournigand $C$ et al. Panitumumab combined with irinotecan for patients with KRAS wild-type metastatic colorectal cancer refractory to standard chemotherapy: a GERCOR efficacy, tolerance, and translational molecular study. Annals of oncology : 
official journal of the European Society for Medical Oncology / ESMO. 2013;24(2):412-9. doi:10.1093/annonc/mds465.

73. Patterson SD, Peeters M, Siena S, Van Cutsem E, Humblet $Y$, Van Laethem JL et al. Comprehensive analysis of KRAS and NRAS mutations as predictive biomarkers for single agent panitumumab (pmab) response in a randomized, phase III metastatic colorectal cancer (mCRC) study (20020408). Journal of clinical oncology : official journal of the American Society of Clinical Oncology. 2013;31 (suppl; abstr 3617).

74. Schwartzberg LS, Rivera F, Karthaus M, Fasola G, Canon JL, Hecht JR et al. PEAK: a randomized, multicenter phase II study of panitumumab plus modified fluorouracil, leucovorin, and oxaliplatin (mFOLFOX6) or bevacizumab plus mFOLFOX6 in patients with previously untreated, unresectable, wildtype KRAS exon 2 metastatic colorectal cancer. Journal of clinical oncology : official journal of the American Society of Clinical Oncology. 2014;32(21):2240-7. doi:10.1200/JCO.2013.53.2473.

75. Peeters $M$, Price TJ, Cervantes A, Sobrero AF, Ducreux $M$, Hotko $Y$ et al. Final results from a randomized phase 3 study of FOLFIRI $\{+/-\}$ panitumumab for second-line treatment of metastatic colorectal cancer. Annals of oncology : official journal of the European Society for Medical Oncology / ESMO. 2014;25(1):107-16. doi:10.1093/annonc/mdt523.

76. Peeters $M$, Oliner KS, Price $T$, Cervantes A, Sobrero AF, Ducreux $M$ et al. Updated analysis of KRAS/NRAS and BRAF mutations in study 20050181 of panitumumab (pmab) plus FOLFIRI for second-line treatment (tx) of metastatic colorectal cancer ( $m C R C)$. Journal of clinical oncology : official journal of the American Society of Clinical Oncology. 2014;32:5s, (suppl; abstr 3568).

77. Sorich MJ, Wiese MD, Rowland A, Kichenadasse G, McKinnon RA, Karapetis CS. Extended RAS mutations and anti-EGFR monoclonal antibody survival benefit in metastatic colorectal cancer: a metaanalysis of randomized, controlled trials. Annals of oncology : official journal of the European Society for Medical Oncology / ESMO. 2015;26(1):13-21. doi:10.1093/annonc/mdu378.

78. Schirripa M, Cremolini C, Loupakis F, Morvillo M, Bergamo F, Zoratto F et al. Role of NRAS mutations as prognostic and predictive markers in metastatic colorectal cancer. International journal of cancer Journal international du cancer. 2015;136(1):83-90. doi:10.1002/ijc.28955.

79. Richman SD, Seymour MT, Chambers P, Elliott F, Daly CL, Meade AM et al. KRAS and BRAF mutations in advanced colorectal cancer are associated with poor prognosis but do not preclude benefit from oxaliplatin or irinotecan: results from the MRC FOCUS trial. Journal of clinical oncology : official journal of the American Society of Clinical Oncology. 2009;27(35):5931-7. doi:10.1200/JCO.2009.22.4295.

80. Stremitzer S, Stift J, Gruenberger B, Tamandl D, Aschacher T, Wolf B et al. KRAS status and outcome of liver resection after neoadjuvant chemotherapy including bevacizumab. The British journal of surgery. 2012;99(11):1575-82. doi:10.1002/bjs.8909.

81. Vauthey JN, Zimmitti G, Kopetz SE, Shindoh J, Chen SS, Andreou A et al. RAS mutation status predicts survival and patterns of recurrence in patients undergoing hepatectomy for colorectal liver metastases. Annals of surgery. 2013;258(4):619-26; discussion 26-7. doi:10.1097/SLA.0b013e3182a5025a.

82. Yaeger R, Cowell E, Chou JF, Gewirtz AN, Borsu L, Vakiani E et al. RAS mutations affect pattern of metastatic spread and increase propensity for brain metastasis in colorectal cancer. Cancer. 2014. doi:10.1002/cncr.29196.

83. Mise Y, Zimmitti G, Shindoh J, Kopetz S, Loyer EM, Andreou A et al. RAS Mutations Predict Radiologic and Pathologic Response in Patients Treated with Chemotherapy Before Resection of Colorectal Liver Metastases. Annals of surgical oncology. 2014. doi:10.1245/s10434-014-4042-6.

84. Wang Y, Carlton VE, Karlin-Neumann G, Sapolsky R, Zhang L, Moorhead M et al. High quality copy number and genotype data from FFPE samples using Molecular Inversion Probe (MIP) microarrays. BMC medical genomics. 2009;2:8. doi:10.1186/1755-8794-2-8. 
85. Srinivasan M, Sedmak D, Jewell S. Effect of fixatives and tissue processing on the content and integrity of nucleic acids. The American journal of pathology. 2002;161(6):1961-71. doi:10.1016/S00029440(10)64472-0.

86. Song S, Nones K, Miller D, Harliwong I, Kassahn KS, Pinese M et al. qpure: A tool to estimate tumor cellularity from genome-wide single-nucleotide polymorphism profiles. PloS one. 2012;7(9):e45835. doi:10.1371/journal.pone.0045835.

87. Atreya CE, Corcoran RB, Kopetz S. Expanded RAS: refining the patient population. Journal of clinical oncology : official journal of the American Society of Clinical Oncology. 2015;33(7):682-5. doi:10.1200/JCO.2014.58.9325.

88. Gerlinger M, Rowan AJ, Horswell S, Larkin J, Endesfelder D, Gronroos E et al. Intratumor heterogeneity and branched evolution revealed by multiregion sequencing. The New England journal of medicine. 2012;366(10):883-92. doi:10.1056/NEJMoa1113205.

89. Diehl F, Schmidt K, Choti MA, Romans K, Goodman S, Li M et al. Circulating mutant DNA to assess tumor dynamics. Nature medicine. 2008;14(9):985-90. doi:10.1038/nm.1789.

90. Board RE, Ellison G, Orr MC, Kemsley KR, McWalter G, Blockley LY et al. Detection of BRAF mutations in the tumour and serum of patients enrolled in the AZD6244 (ARRY-142886) advanced melanoma phase II study. British journal of cancer. 2009;101(10):1724-30. doi:10.1038/sj.bjc.6605371.

91. Lefebure B, Charbonnier F, Di Fiore F, Tuech JJ, Le Pessot F, Michot F et al. Prognostic value of circulating mutant DNA in unresectable metastatic colorectal cancer. Annals of surgery. 2010;251(2):27580. doi:10.1097/SLA.0b013e3181c35c87.

92. Chen H, Tu H, Meng ZQ, Chen Z, Wang P, Liu LM. K-ras mutational status predicts poor prognosis in unresectable pancreatic cancer. European journal of surgical oncology : the journal of the European Society of Surgical Oncology and the British Association of Surgical Oncology. 2010;36(7):657-62. doi:10.1016/j.ejso.2010.05.014.

93. Yen LC, Yeh YS, Chen CW, Wang HM, Tsai HL, Lu CY et al. Detection of KRAS oncogene in peripheral blood as a predictor of the response to cetuximab plus chemotherapy in patients with metastatic colorectal cancer. Clinical cancer research : an official journal of the American Association for Cancer Research. 2009;15(13):4508-13. doi:10.1158/1078-0432.CCR-08-3179.

94. Barbazan J, Muinelo-Romay L, Vieito M, Candamio S, Diaz-Lopez A, Cano A et al. A multimarker panel for circulating tumor cells detection predicts patient outcome and therapy response in metastatic colorectal cancer. International journal of cancer Journal international du cancer. 2014;135(11):2633-43. doi:10.1002/ijc.28910.

95. Mostert B, Jiang $\mathrm{Y}$, Sieuwerts AM, Wang $\mathrm{H}$, Bolt-de Vries J, Biermann $\mathrm{K}$ et al. KRAS and BRAF mutation status in circulating colorectal tumor cells and their correlation with primary and metastatic tumor tissue. International journal of cancer Journal international du cancer. 2013;133(1):130-41. doi:10.1002/ijc.27987.

96. van Krieken JH, Jung A, Kirchner T, Carneiro F, Seruca R, Bosman FT et al. KRAS mutation testing for predicting response to anti-EGFR therapy for colorectal carcinoma: proposal for an European quality assurance program. Virchows Archiv : an international journal of pathology. 2008;453(5):417-31. doi:10.1007/s00428-008-0665-y.

97. Forshew T, Murtaza M, Parkinson C, Gale D, Tsui DW, Kaper F et al. Noninvasive identification and monitoring of cancer mutations by targeted deep sequencing of plasma DNA. Science translational medicine. 2012;4(136):136ra68. doi:10.1126/scitranslmed.3003726.

98. Cancer Genome Atlas N. Comprehensive molecular characterization of human colon and rectal cancer. Nature. 2012;487(7407):330-7. doi:10.1038/nature11252. 
Figure 1. EGFR pathway. 1) Normal state, the EGFR pathway has been presented upon binding of a ligand to EGFR; 2) the EGFR pathway is blocked by anti-EGFR therapy, which inhibits cell proliferation and survival; 3) a mutation in RAS (star) causes the constitutive activation of the EGFR pathway resulting in cell proliferation and survival, despite the blocking of EGFR by anti-EGFR therapy. 